\title{
Orthogonal tensor decomposition and orbit closures from a linear algebraic perspective
}

\author{
Pascal Koiran*
}

October 1, 2019

\begin{abstract}
We study orthogonal decompositions of symmetric and ordinary tensors using methods from linear algebra. For the field of real numbers we show that the sets of decomposable tensors can be defined by equations of degree 2. This gives a new proof of some of the results of Robeva and Boralevi et al. Orthogonal decompositions over the field of complex numbers had not been studied previously; we give an explicit description of the set of decomposable tensors using polynomial equalities and inequalities, and we begin a study of their closures.

The main open problem that arises from this work is to obtain a complete description of the closures. This question is akin to that of characterizing border rank of tensors in algebraic complexity. We give partial results using in particular a connection with approximate simultaneous diagonalization (the so-called $A S D$ property).

Keywords: tensor decomposition, tensor rank, orbit closure, Waring decomposition, border rank.
\end{abstract}

\footnotetext{
${ }^{*}$ Univ Lyon, EnsL, UCBL, CNRS, LIP, F-69342, LYON Cedex 07, France. Email: pascal.koiran@ens-lyon.fr. This work got started at the Simons Institute for the Theory of Computing during the Fall 2018 program on Lower Bounds in Computational Complexity.
} 


\section{Contents}

1 Introduction 3

1.1 Results and methods . . . . . . . . . . . . . . . . 4

1.2 Orbit closures . . . . . . . . . . . . . . . . . 5

1.3 Algorithmic issues . . . . . . . . . . . . . . . . . 6 6

1.4 Open problems ........................

2 Background 7

2.1 Tensors and their slices . . . . . . . . . . . . . . 7

2.2 Diagonalization and constructible sets . . . . . . . . 10

2.3 Simultaneous diagonalization . . . . . . . . . . . . . 10

2.4 Quadratic forms .................... 11

3 Orthogonal Waring decomposition 14

3.1 Orthogonal Waring decomposition over the reals . . . . . 15

3.2 Orthogonal Waring decomposition over the complex numbers 17

3.3 Closure properties from below . . . . . . . . . . . . . 18

3.4 The ASD property . . . . . . . . . . . . . . . 24

4 Orthogonal decomposition of ordinary tensors 30

4.1 Orthogonal decomposition of real tensors . . . . . . . . . . 31

4.2 Orthogonal decomposition of complex tensors . . . . . . . . 34

4.3 Closure properties . . . . . . . . . . . . . . . . 36 


\section{Introduction}

In this paper we study several types of orthogonal tensor decompositions and give algebraic characterizations of the set of decomposable tensors. That is, we give explicit systems of polynomial equations whose zero set is the set of decomposable tensors. When that is not possible, i.e., when the set of decomposable tensors is not Zariski closed, we describe it as a constructible set (using polynomial inequalities in addition to polynomial equalities). In the non-closed case we begin a study of the closure. The main goal here would be to obtain an explicit description of the closure, and we give partial results in this direction.

The decompositions that we study can be defined in two equivalent languages: the language of tensors, and the language of polynomials. Indeed, as is well known one can associate to a symmetric tensor (respectively, to an ordinary tensor) a homogeneous polynomial (respectively, a multilinear polynomial) in the same way that a quadratic form is associated to a symmetric matrix and a bilinear form is associated to an arbitrary matrix. We begin with a definition in the language of polynomials, but we will switch between the two languages whenever that is convenient.

Let $K$ be a field of characteristic 0 . We denote by $K\left[x_{1}, \ldots, x_{n}\right]_{d}$ the space of homogeneous polynomials of degree $d$ in $n$ variables (also called: "degree $d$ forms"). The cases of interest for this paper are $K=\mathbb{R}$ and $K=\mathbb{C}$. Recall that a matrix $A \in M_{n}(K)$ is said to be orthogonal if $A^{T} A=\operatorname{Id}_{n}$.

Definition 1. We say that $f \in K\left[x_{1}, \ldots, x_{n}\right]_{d}$ admits an orthogonal Waring decomposition if it can be written as $f(x)=g(A x)$ where $A$ is an orthogonal matrix and $g$ is any polynomial of the form

$$
g\left(x_{1}, \ldots, x_{n}\right)=\alpha_{1} x_{1}^{d}+\cdots+\alpha_{n} x_{n}^{d}
$$

with $\alpha_{1}, \ldots, \alpha_{n} \in K$.

In this paper we focus on the case $d=3$, which corresponds to symmetric tensors of order 3. We will denote by $\mathrm{OW}_{n}(K)$ the set of homogeneous polynomials of degree 3 in $n$ variables that admit such a decomposition, and we will identify it with the corresponding set of symmetric tensors.

For $K=\mathbb{R}$, Definition 1 turns out to be equivalent to the notion of symmetrically odeco tensor studied in [5]. According to [5], a symmetric tensor is symmetrically odeco if it can be written as

$$
\sum_{i=1}^{k} \alpha_{i} v_{i}^{\otimes d}
$$

where $\alpha_{i}= \pm 1$ and $v_{1}, \ldots, v_{k}$ are nonzero, pairwise orthogonal vectors in $\mathbb{R}^{n}$.

Let us now move to ordinary tensors. As recalled above, an ordinary tensor of order three $T \in K^{n \times n \times n}$ can be represented by a trilinear form 
$t(x, y, z)=\sum_{i, j, k=1}^{n} T_{i j k} x_{i} y_{j} z_{k}$ where $x, y, z$ denote three $n$-tuples of variables.

Definition 2. We say that the trilinear form $t(x, y, z) \in K[x, y, z]$, or the corresponding tensor $T$, admits an orthogonal decomposition if one can write $t(x, y, z)=g(A x, B y, C z)$ where $A, B, C$ are orthogonal matrices and $g$ is a diagonal trilinear form in $3 n$ variables, i.e., a polynomial of the form:

$$
g(x, y, z)=\sum_{i=1}^{n} \alpha_{i} x_{i} y_{i} z_{i}
$$

with $\alpha_{1}, \ldots, \alpha_{n} \in K$.

We will denote by $\mathrm{OT}_{n}(K)$ the set of trilinear forms that admit an orthogonal decomposition, and we will use the same notation for the corresponding set of tensors. For $K=\mathbb{R}$, it turns out that Definition 2 agrees with the definition of an "odeco tensor" from [5]: an order 3 tensor is odeco if it can be written as

$$
\sum_{i=1}^{k} u_{i} \otimes v_{i} \otimes w_{i}
$$

where each of the the 3 lists $\left(u_{1}, \ldots, u_{k}\right),\left(v_{1}, \ldots, v_{k}\right),\left(w_{1}, \ldots, w_{k}\right)$ is made of $k$ nonzero, pairwise orthogonal vectors in $\mathbb{R}^{n}$.

\subsection{Results and methods}

A tensor $T$ of order 3 and size $n$ can be viewed as $n$ matrices (the "slices" of $T$ ) stacked on top of each other. This very down-to-earth point of view turns out to be remarkably powerful for the study of orthogonal decompositions because it allows us to leverage the known body of work on simultaneous reduction of matrices. For some of our results about the complex field we also need elements of the theory of quadratic forms (and in particular the notions of isotropic vectors, totally isotropic subspaces, Witt's extension theorem...1). Our contributions are twofold:

(i) We give more elementary proofs of two results from [5]: the set of odeco and symmetrically odeco tensors can be described by equations of degree 2. In [5] the result for symmetrically odeco tensors is obtained as follows: given a symmetric tensor $S$ of order 3, they define a bilinear map from $\mathbb{R}^{n} \times \mathbb{R}^{n}$ to $\mathbb{R}^{n}$ associated to $S$ in a natural way; then they show that $S$ is symmetrically odeco iff this map gives rise to an associative algebra. We obtain an alternative, intuitively appealing characterization: $S$ is symmetrically odeco iff its slices commute. We give two different proofs of this fact (see Section 3.1 for details).

\footnotetext{
${ }^{1}$ As a side remark, isotropic subspaces also appear in the recent paper [3] where a connection is made to graph-theoretic concepts such as the chromatic number and the independence number.
} 
(ii) We initiate a study of orthogonal decomposition over the field of complex numbers, which up to now had not been studied either from an algorithmic or structural point of view. In particular, we stress that all the results in [5] for the field of complex numbers are obtained for unitary rather than orthogonal decompositions.

In this paper we give characterizations of the set of symmetric and ordinary tensors that admit orthogonal decompositions in the sense of Definitions 1 and 2, In particular, for orthogonal Waring decomposition over $\mathbb{C}$ the slices must commute and be diagonalizable.

Compared to unitary or real orthogonal decompositions, a distinctive feature of complex orthogonal decompositions, and our main motivation for studying them, is that the set of decomposable tensors is not closed (as could be guessed from the diagonalizability condition in the above characterization). We elaborate on this in Section 1.2 .

A remark is in order regarding the above point (i). Jan Draisma (personal communication) has pointed out that the authors of [5] first worked in the language of slices before switching to the language of algebras, which turned out to be more efficient for their purposes. It may still be useful to present the first point of view in detail here since there does not remain any trace of it in [5. More importantly, this point of view seems better suited to the study of complex orthogonal decompositions because, as explained in (ii), the sets of decomposable tensors are not closed.

\subsection{Orbit closures}

According to Definition 1, the set of decomposable tensors is the orbit of the set of polynomials of the form (11) under the action of the orthogonal group. The closure of the set of decomposable tensors is therefore an orbit closure. We have a similar situation in Definition 2 with the action of a product of 3 orthogonal groups. The notion of orbit closure plays a central in Mulmuley and Sohoni's Geometric Complexity Theory [19, 20]. In their program, the goal is to show that the permanent polynomia 2 is not in the orbit closure of the determinant (under the action of the general linear group).

Closer to the topic of the present paper, we find the notion of border rank of tensors which plays an important role in the the study of matrix multiplication algorithms [7. If we replace in Definition 2 the orthogonal group by the general linear group, the corresponding orbit closure is the set of tensors of border rank at most $n$. The same change in Definition 1 would yield the set of polynomials of border Waring rank at most $n$. Restricting to the orthogonal group as we do in this paper yields orbit closures which should hopefully be easier to study. We study them "from below", i.e., we

\footnotetext{
${ }^{2}$ More precisely, a "padded" version of the permanent.
} 
find nontrivial families of tensors which belong to the orbit closures; and we study them "from above", i.e., we find equations that must be satisfied by all tensors in the orbit closures. For instance, we show that the slices of any tensor in the closure $\overline{\mathrm{OW}_{n}(\mathbb{C})}$ of $\mathrm{OW}_{n}(\mathbb{C})$ must commute, that they generate a matrix algebra of dimension at most $n$, and that their centralizer is of dimension at least $n$. We obtain these equations thanks to a connection with the so-called "ASD property" [22, 21]:

Definition 3. A tuple $\left(A_{1}, \ldots, A_{k}\right)$ of matrices in $M_{n}(\mathbb{C})$ is approximately simultaneously diagonalizable ( $A S D$ for short) if it is in the closure of the set of $k$-tuples of simultaneously diagonalizable matrices, i.e., if for any $\epsilon>0$ there exist simultaneously diagonalizable matrices $B_{1}, \ldots, B_{k}$ which satisfy $\left\|A_{i}-B_{i}\right\|<\epsilon$ for $i=1, \ldots, k$.

Indeed, we show that the slices of a tensor in $\overline{\mathrm{OW}_{n}(\mathbb{C})}$ must satisfy the ASD property.

\subsection{Algorithmic issues}

The characterizations that we obtain in our 4 main scenarios (orthogonal Waring decomposition of symmetric tensors and orthogonal decomposition of ordinary tensors, over the real and complex fields) are straightfoward to check algorithmically: they involve only standard linear-algebraic computations on the slices of the tensors (as a previously mentioned example, for orthogonal Waring decomposition over $\mathbb{C}$ the slices must commute and be diagonalizable). From these characterizations one could also derive algorithms that effectively construct a decomposition whenever that is possible. These algorithms would rely on standard routines from linear algebra such as simultaneous matrix diagonalization. We will not go into the details in the present paper but we note that there is a large literature on algorithms for various types of tensor decompositions (see e.g. [2, 16, 25, 27]), and that they are often based on linear algebra. In particular, the preprint [16] makes the connection between simultaneous diagonalization and orthogonal decomposition of real symmetric tensors. Therefore, one contribution of our paper is to show that techniques from linear algebra are not only useful for designing decomposition algorithms, but also to obtain algebraic characterizations 3

\subsection{Open problems}

The main open problem that arises from this work is the complete determination of the orbit closures $\overline{\mathrm{OW}_{n}(\mathbb{C})}$ and $\overline{\mathrm{OT}_{n}(\mathbb{C})}$. Indeed, as shown later

\footnotetext{
${ }^{3}$ We note that the connection between singular value decomposition and tensor decomposition algorithms is made in [5] (see Proposition 7 from that paper, and the remarks thereafter). However, the algebraic characterizations obtained later in [5] do not rely on the SVD or similar techniques.
} 
in the paper the study of the orbit closures "from below" and "from above" do not lead to a complete characterization. This question bears a certain similarity to another open problem at the intersection of linear algebra and algebraic geometry: obtaining a complete characterization of the ASD property [22, 21]. For application to orthogonal tensor decompositions, we note that it suffices to study the ASD property for tuples of symmetric matrices, and furthermore to consider only approximations by tuples of simultaneously diagonalizable symmetric matrices. This holds true even for ordinary tensors, see Proposition 67 and Remark 68 at the end of the paper.

As explained in Section 1.2, if the orthogonality requirement is lifted from our tensor decompositions one obtains instead of $\overline{\mathrm{OW}_{n}(\mathbb{C})}$ and $\overline{\mathrm{OT}_{n}(\mathbb{C})}$ the sets of tensors of border (Waring) rank at most $n$. Obtaining explicit equations for these sets is vey much an open problem, with applications to lower bounds in algebraic complexity theory (see e.g. [17]).

In this paper we have studied decompositions of order 3 tensors. In [5], tensors of higher order are handled by reduction to the case of order 3 . Namely, they show that a tensor of order $d \geq 4$ admits a unitary or real orthogonal decomposition iff the same is true for certain flattenings of the tensor. It would be interesting to find out whether a similar property holds for complex orthogonal decompositions. Following this approach would also require a generalization of our results to "rectangular" tensors of order 3. Indeed, we have only studied "cubic" tensors (of format $n \times n \times n$ ). But even if we start from a higher order cubic tensor (e.g., a tensor $T$ of order 4 and format $n \times n \times n \times n$ ), its flattenings will not be cubic in general. For instance, we would obtain from $T$ flattenings of format $n \times n \times n^{2}$.

\section{Background}

In this section we first present some background on tensors and matrices. Indeed, as explained in the introduction simultaneous reduction of matrices (and in particular simulataneous diagonalization) plays an important role in this paper. For the study of complex tensors we will also need some elements of the theory of quadratic forms, which we present in Section 2.4.

\subsection{Tensors and their slices}

An order 3 tensor $T \in K^{n \times n \times n}$ can be represented by the trilinear form

$$
t(x, y, z)=\sum_{i, j, k=1}^{n} T_{i j k} x_{i} y_{j} z_{k}
$$

where $x, y, z$ denote three $n$-tuples of variables. There are 3 ways of decomposing $T$ into a tuple of $n$ matrices: we can decompose in the $x, y$ 
or $z$ direction. We call the resulting matrices the $x$-slices, $y$-slices, and $z$ slices. For instance, the $z$-slices are the matrices of the bilinear forms $\partial t / \partial z_{k}$ $(1 \leq k \leq n)$.

The tensor $T$ is said to be symmetric if it is invariant under all of the six permutations of the indices $i, j, k$. For such a tensor the $x, y$ and $z$ slices are identical and we simply call them "the $n$ slices of $T$ "; each of these slices is a symmetric matrix. To a symmetric tensor $T$ we associate the degre 3 form $f\left(x_{1}, \ldots, x_{n}\right)=\sum_{i, j, k=1}^{n} T_{i j k} x_{i} x_{j} x_{k}$. Note that $f$ is obtained from the trilinear form $t$ in (3) by setting $x=y=z$, in the same way that a quadratic form is obtained from a bilinear form. Since we switch freely between the language of tensors and the language of polynomials (for slices and for other notions), by "slices of $f$ " we will mean the slices of the corresponding symmetric tensor $T$.

In light of Definitions 1 1and 2, it is important to understand how slices are affected by a linear change of variables. Let us do this for symmetric tensors: we will show that the slices $S_{1}, \ldots, S_{n}$ of a symmetric tensor $S \in \mathrm{OW}_{n}(K)$ are given by:

$$
S_{k}=A^{T} D_{k} A, D_{k}=\operatorname{diag}\left(\alpha_{1} a_{1 k}, \ldots, \alpha_{n} a_{n k}\right) .
$$

Our proof will use the following property of Hessian matrices ([13], Lemma $5.1)$.

Fact 1. Let $G$ be an $n$-variate polynomial and $A \in K^{n \times n}$ a linear transformation. Let $F(x)=G(A x)$. The Hessian matrices of $F$ and $G$ satisfy the relation:

$$
H_{F}(x)=A^{T} H_{G}(A x) A .
$$

Consider any homogeneous polynomial of degree 3 . The entries of $H_{f}$ are linear forms in $x_{1}, \ldots, x_{n}$. One can therefore write $H_{f}=x_{1} A_{1}+\cdots+x_{n} A_{n}$ where the $A_{i}$ are $n \times n$ matrices with entries in $K$. These matrices are symmetric since entry $(j, k)$ of $A_{i}$ is given by:

$$
\left(A_{i}\right)_{j k}=\frac{\partial^{3} f}{\partial x_{i} \partial x_{j} \partial x_{k}} .
$$

Each entry of $A_{i}$ therefore corresponds to a monomial of $f$. More precisely, $\left(A_{i}\right)_{j k}$ is obtained by multiplication of the coefficient of $x_{i} x_{j} x_{k}$ by 1,2 or 6 . Equivalently, one can define $A_{i}$ as the Hessian matrix of $\partial f / \partial x_{i}$.

Remark 4. It follows from (6) that the $A_{i}$ are scalar multiples of the slices of the symmetric tensor associated to $f$, and the constant of proportionality is equal to 6 .

We illustrate this point on the example of the polynomial $f\left(x_{1}, x_{2}\right)=$ $\left(x_{1}-x_{2}\right)^{3}$. The corresponding tensor is the rank 1 symmetric tensor $T=e^{\otimes 3}$ where $e=(1,-1)$. The entries of $T$ are: $T_{111}=1, T_{112}=-1, T_{122}=1$ and 
$T_{222}=-1$ (the other entries are obtained from these 4 entries by symmetry of $T)$. The two slices of $T$ are the matrices:

$$
T_{1}=\left(\begin{array}{cc}
1 & -1 \\
-1 & 1
\end{array}\right), T_{2}=-T_{1}
$$

and it is easy to check that the Hessian matrices of $\partial f / \partial x_{1}, \partial f / \partial x_{2}$ are respectively $6 T_{1}$ and $6 T_{2}$.

Lemma 5. The Hessian matrix of a polynomial $g \in K\left[x_{1}, \ldots, x_{n}\right]_{3}$ is diagonal if and only if $g$ is of the form (11).

Proof. If $g$ is of the required form, $H_{g}=\operatorname{diag}\left(6 \alpha_{1} x_{1}, \ldots, 6 \alpha_{n} x_{n}\right)$. Conversely, if $H_{g}$ is diagonal, $g$ can only contain cubes of powers since any other monomial would give rise to an off-diagonal term in $H_{g}$.

We can now establish formula (4):

Proposition 6. The slices $S_{1}, \ldots, S_{n}$ of a polynomial $f \in K\left[x_{1}, \ldots, x_{n}\right]_{3}$ as in Definition 1 are given by (4).

Proof. The Hessian matrix of the polynomial $g=\alpha_{1} x_{1}^{3}+\ldots+\alpha_{n} x_{n}^{3}$ is $H_{g}=\operatorname{diag}\left(6 \alpha_{1} x_{1}, \ldots, 6 \alpha_{n} x_{n}\right)$. Hence the result follows from Fact 1 since $A_{k}=6 S_{k}$.

We will establish a similar result for ordinary tensors in Proposition 50 , In particular, we will show (following the notations of Definition 2) that the $z$-slices $T_{1}, \ldots, T_{n}$ of a trilinear form $t \in \mathrm{OT}_{n}(K)$ are given by the formula

$$
T_{k}=A^{T} D_{k} B, D_{k}=\operatorname{diag}\left(\alpha_{1} c_{1 k}, \ldots, \alpha_{n} c_{n k}\right)
$$

where the $c_{i k}$ are the entries of $C$. Note that (4) can also be derived from this formula. Consider indeed a degree 3 form $f \in \mathrm{OW}_{n}(K)$. By Definition 1 , $f(x)=h(x, x, x)$ where $h$ is the trilinear form $g(A x, A y, A z)$ and $g$ is as in (2). Viewed as an ordinary tensor, a symmetric tensor in $\mathrm{OW}_{n}(K)$ therefore belongs to $\mathrm{OT}_{n}(K)^{4}$ and we can obtain (4) by setting $A=B=C$ in (17).

Finally, we mention a consequence of Fact 1 (Lemma 5.2 in [13]) which will be useful in Section 3.3

Lemma 7. Let $f \in K\left[x_{1}, \ldots, x_{n}\right]$ be a polynomial of the form

$$
f\left(x_{1}, \ldots, x_{n}\right)=\sum_{i=1}^{n} a_{i} \ell_{i}\left(x_{1}, \ldots, x_{n}\right)^{d}
$$

\footnotetext{
${ }^{4}$ As a sanity check, one can easily verify that if a symmetric tensor satisfies the conditions for membership in $\mathrm{OW}_{n}(\mathbb{R})$ (Theorem 20) or $\mathrm{OW}_{n}(\mathbb{C})$ (Theorem 23) then it also satisfies the conditions for membership in $\mathrm{OT}_{n}(\mathbb{R})($ Theorem 55$)$ or $\mathrm{OT}_{n}(\mathbb{C})$ (Theorem 59).
} 
where the $\ell_{i}$ are linearly independent linear forms, the $a_{i}$ are nonzero constants and $d \geq 2$. Then the Hessian determinant of $f$ satisfies

$$
\operatorname{det} H_{f}\left(x_{1}, \ldots, x_{n}\right)=c \prod_{i=1}^{n} \ell_{i}\left(x_{1}, \ldots, x_{n}\right)^{d-2}
$$

where $c \in K$ is a nonzero constant. In particular, $\operatorname{det} H_{f}$ is not identically 0 .

\subsection{Diagonalization and constructible sets}

One of the main goals of the paper is to give necessary and sufficient conditions for membership of symmetric tensors in $\mathrm{OW}_{n}(\mathbb{C})$ and of ordinary tensors in $\mathrm{OT}_{n}(\mathbb{C})$. In particular, certain matrices (slices, or products of slices) must be diagonalizable. Since the set of diagonalizable matrices is dense in $M_{n}(\mathbb{C})$, it cannot be written as the zero set of of a system of polynomial equations. It can however be described by polynomial equalities and inequalities, i.e., it is a constructible subset of $M_{n}(\mathbb{C})$. This follows from the following well known result:

Proposition 8. Let $K$ be a field of characteristic 0 and let $\chi_{M}$ be the characteristic polynomial of a matrix $M \in M_{n}(K)$. Let $P_{M}=\chi_{M} / \operatorname{gcd}\left(\chi_{M}, \chi_{M}^{\prime}\right)$ be the squarefree part of $\chi_{M}$. The matrix $M$ is diagonalizable over $\bar{K}$ iff $P_{M}(M)=0$.

Proposition 8 together with the characterizations in Sections 3.2 and 4.2 show that the sets of tensors $\mathrm{OW}_{n}(\mathbb{C})$ and $\mathrm{OT}_{n}(\mathbb{C})$ are constructible.

If we restrict to symmetric matrices, it is still true that diagonalizable matrices are dense. This fact will be used in Section 3.3. For the sake of completeness we give the (standard) proof below.

Lemma 9. The set of diagonalizable symmetric matrices is dense in the set of complex symmetric matrices.

Proof. We will prove a stronger result: the set of symmetric matrices with $n$ distinct eigenvalues is dense in the set of symmetric matrices of size $n$. In order to show this we associate to a symmetric matrix $S$ of size $n$ the discriminant $\operatorname{Disc}_{S}$ of its characteristic polynomial. Our matrix has $n$ distinct eigenvalues if and only if $\operatorname{Disc}_{S} \neq 0$. Note that this discriminant can be viewed as a polynomial in the $n(n+1) / 2$ upper triangular entries of $S$. Therefore, the conclusion will follow if we can show that this polynomial

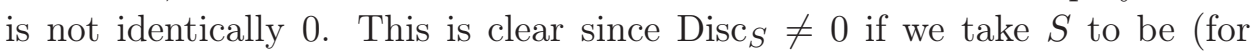
instance) a diagonal matrix with distinct diagonal entries.

\subsection{Simultaneous diagonalization}

As mentioned in the introduction several of our results hinge on simultaneous reduction of matrices, and in particular on simultaneous diagonalization. 
Lemma 10. Let $A_{1}, \ldots, A_{k} \in M_{n}(K)$ be a tuple of simultaneously diagonalizable matrices, and let $S \subseteq K$ be a finite set of size $|S|>n(n-1) / 2$. Then there exist $\alpha_{2}, \ldots, \alpha_{k}$ in $S$ such that any transition matrix which diagonalizes $A_{1}+\alpha_{2} A_{2}+\ldots+\alpha_{k} A_{k}$ must also diagonalize all of the matrices $A_{1}, \ldots, A_{k}$.

Proof. We proceed by induction on $k$. The base case $k=2$ is Proposition 2 in [14. Assume now that the result holds true at step $k-1$. By induction hypothesis there exist $\alpha_{2}, \ldots, \alpha_{k-1}$ in $S$ such that any transition matrix which diagonalizes $M=A_{1}+\alpha_{2} A_{2}+\ldots+\alpha_{k-1} A_{k-1}$ must also diagonalize $A_{1}, \ldots, A_{k-1}$. Moreover, by the base case there exists $\alpha_{k} \in S$ such that any transition matrix $T$ which diagonalizes $M+\alpha_{k} A_{k}$ must diagonalize $M$ and $A_{k}$. Therefore such a $T$ must diagonalize all of the $A_{1}, \ldots, A_{k}$.

This lemma has the following important consequence.

Theorem 11. Let $A_{1}, \ldots, A_{k}$ be a tuple of symmetric matrices of $M_{n}(K)$ where $K=\mathbb{R}$ or $K=\mathbb{C}$. If the $A_{i}$ are simultaneously diagonalizable then they are simultaneously diagonalizable by an orthogonal change of basis, i.e., there is a real (respectively, complex) orthogonal matrix $P$ such that the $k$ matrices $P^{T} A_{i} P$ are diagonal.

Proof. We begin with $K=\mathbb{R}$. Let us fix $\alpha_{2}, \ldots, \alpha_{k}$ as in the previous lemma. Since the matrix $S=A_{1}+\alpha_{2} A_{2}+\ldots+\alpha_{k} A_{k}$ is real symmetric, it can be diagonalized by a real orthogonal matrix. By the lemma, such a matrix will diagonalize all of the $A_{i}$.

For $K=\mathbb{C}$, the difference with the real case is that it is no longer true that all symmetric matrices are diagonalizable (see e.g. Example 29] and the beginning of Section 3.25). It is however still the case that if a complex symmetric matrix is diagonalizable, then it can be diagonalized by an orthogonal matrix (Theorem 4.4.27 of [12]). In the present situation, $S$ will be diagonalizable for any choice of complex numbers $\alpha_{2}, \ldots, \alpha_{k}$ since the $A_{i}$ are simultaneously diagonalizable. The result therefore follows from Lemma 10 like in the real case.

It is possible to give a direct (non inductive) proof of this theorem based on Theorem 6.4.16, Corollary 6.4.18 and Corollary 6.4.19 of [11]. Moreover, this argument shows that the conclusion of Theorem 111 holds not only when the $A_{i}$ are symmetric, but also when they are all skew-symmetric or all orthogonal (Roger Horn, personal communication).

\subsection{Quadratic forms}

As mentioned before, for $K=\mathbb{C}$ we need some elements of the theory of quadratic forms. We will work only with the quadratic form $\sum_{i=1}^{n} x_{i}^{2}$ on $\mathbb{C}^{n}$

\footnotetext{
${ }^{5}$ The existence of such examples is due to the presence of isotropic vectors in $\mathbb{C}^{n}$; more on this topic in Section 2.4.
} 
and the associated bilinear map $\langle x, y\rangle=\sum_{i=1}^{n} x_{i} y_{i}$ but much of what follows applies to an arbitrary nondegenerate quadratic space. We will refer to this bilinear map as the "Euclidean inner product", but this is an abuse of terminology since we are working over the field of complex numbers. Compared to the case $K=\mathbb{R}$ the main complication (and the reason why one often works with Hermitian and unitary matrices rather than symmetric and orthogonal matrices) is that $\mathbb{C}^{n}$ contains isotropic vectors.

Definition 12. A vector $v \in \mathbb{C}^{n} \backslash\{0\}$ is isotropic if it is self-orthogonal, i.e., if $\langle v, v\rangle=0$.

More generally, a subspace $V \subseteq \mathbb{C}^{n}, V \neq\{0\}$ is said to be totally isotropic if the Euclidean inner product is identically 0 on $V$, or equivalently if $V$ contains only isotropic vectors.

Whether the null vector is defined to be isotropic or not is a matter of convention.

Theorem 13. Let $U$ be a totally isotropic subspace of $\mathbb{C}^{n}$ with basis $\left(u_{1}, \ldots, u_{r}\right)$. There exists another totally isotropic subspace $U^{\prime}$, disjoint from $U$, with basis $\left(u_{1}^{\prime}, \ldots, u_{r}^{\prime}\right)$ such that $\left\langle u_{i}, u_{j}^{\prime}\right\rangle=\delta_{i j}$.

This lemma applies not only to $\mathbb{C}^{n}$ but to any nondegenerate quadratic space. For a proof see Theorem 6.2 in the lecture notes [9], where $U^{\prime}$ is called an "isotropic supplement" to $U$.

Corollary 14. Let $\left(u_{1}, \ldots, u_{k}\right)$ be a tuple of linearly independent pairwise orthogonal vectors of $\mathbb{C}^{n}$. If there are $r$ isotropic vectors in this tuple then $r+k \leq n$. In particular, if $U$ is a totally isotropic subspace of $\mathbb{C}^{n}$ then $\operatorname{dim} U \leq n / 2$.

Proof. Assume for instance that $u_{1}, \ldots, u_{r}$ are the isotropic vectors in this tuple. Let $U$ be the (totally isotropic) subspace spanned by $u_{1}, \ldots, u_{r}$ and let $V$ be the subspace spanned by the non-isotropic vectors $u_{r+1}, \ldots, u_{k}$. Let $U^{\prime}$ be the "isotropic supplement" to $U$ provided by Theorem 13. We claim that $U^{\prime}$ is disjoint from $U \oplus V$. Suppose indeed that $u^{\prime}=u+v$ with $u^{\prime} \in U^{\prime}$, $u \in U, v \in V$. Let us write $u^{\prime}$ as $u^{\prime}=\sum_{j=1}^{r} \alpha_{j} u_{j}^{\prime}$ where $\left(u_{1}^{\prime}, \ldots, u_{r}^{\prime}\right)$ is the basis of $U^{\prime}$ provided by Theorem 13, For any $i \leq r$ we have $\left\langle u^{\prime}, u_{i}\right\rangle=\alpha_{i}$ since $\left\langle u_{i}, u_{j}^{\prime}\right\rangle=\delta_{i j}$. On the other hand, we have

$$
\left\langle u^{\prime}, u_{i}\right\rangle=\left\langle u+v, u_{i}\right\rangle=\left\langle u, u_{i}\right\rangle+\left\langle v, u_{i}\right\rangle=0 .
$$

In the last equality we have used the fact that $\left\langle u, u_{i}\right\rangle=0$ (since $U$ is totally isotropic) and $\left\langle v, u_{i}\right\rangle=0$ (since $u_{1}, \ldots, u_{k}$ are pairwise orthogonal). This proves the claim since we have shown that $\alpha_{i}=0$ for all $i$, i.e., $u^{\prime}=0$.

The conclusion of Corollary 14 follows directly from the claim: we have $U^{\prime} \oplus U \oplus V \subseteq \mathbb{C}^{n}$ so $\operatorname{dim} U+\operatorname{dim} V+\operatorname{dim} U^{\prime} \leq n ;$ but $\operatorname{dim} U=\operatorname{dim} U^{\prime}=r$ and $\operatorname{dim} V=k-r$. 
We will also use the following version of Witt's extension theorem, a cornerstone of the theory of quadratic forms.

Theorem 15. Let $U$ be a linear subspace of $\mathbb{C}^{n}$. Any isometric embedding $\phi: U \rightarrow \mathbb{C}^{n}$ extends to an isometry $F$ of $\mathbb{C}^{n}$.

Here, "isometric embedding" means that $\phi$ is an injective linear map which preserves the Euclidean inner product. Likewise, an isometry of $\mathbb{C}^{n}$ is a linear automorphism which respects the inner product (i.e., $F$ is represented in the standard basis by an orthogonal matrix). Witt's theorem applies not only to $\mathbb{C}^{n}$ but to any nondegenerate quadratic space. For a proof see e.g. [23. Theorem 42:17] or [26, Theorem 5.3 of Chapter 1] or Corollary 7.4 in the lecture notes [9].

Corollary 16. Let $v_{1}, \ldots, v_{k}$ be an orthornormal family of vectors of $\mathbb{C}^{n}$ (i.e., $\left\langle v_{i}, v_{j}\right\rangle=\delta_{i j}$ ). This family can be extended to an orthonormal basis $v_{1}, \ldots, v_{n}$ of $\mathbb{C}^{n}$.

Proof. Let $\left(e_{1}, \ldots, e_{n}\right)$ be the standard basis of $\mathbb{C}^{n}$ and let $U$ be the subspace spanned by $e_{1}, \ldots, e_{k}$. The family $v_{1}, \ldots, v_{k}$ is linearly independent since it is orthonormal. As a result, there is a (unique) isometric embedding $\phi: U \rightarrow \mathbb{C}^{n}$ such that $\phi\left(e_{i}\right)=u_{i}$ for $i=1, \ldots, k$. Let $F$ be the isometry of $\mathbb{C}^{n}$ provided by Theorem [15. The desired orthonormal basis is $v_{1}=$ $F\left(e_{1}\right), \ldots, v_{n}=F\left(e_{n}\right)$.

Here is another useful consequence of Witt's theorem:

Corollary 17. Let $\left(u_{1}, \ldots, u_{k}\right)$ be a tuple of linearly independent pairwise orthogonal vectors of $\mathbb{C}^{n}$. Assume that $\left(u_{1}, \ldots, u_{r}\right)$ are the isotropic vectors in this list, and denote by $U$ the subspace that they span.

The "isotropic supplement" to U provided by Theorem 19 can be chosen to be orthogonal to the subspace $V$ spanned by $\left(u_{r+1}, \ldots, u_{k}\right)$.

Proof. Let $\left(e_{1}, \ldots, e_{n}\right)$ be the standard basis of $\mathbb{C}^{n}$ and consider the vectors $w_{1}, \ldots, w_{k}$ defined as follows: $w_{j}=e_{2 j-1}+i e_{2 j}$ for $j \leq r$ and $w_{j}=e_{r+j}$ for $r+1 \leq j \leq k$. These vectors are well defined since $k+r \leq n$ according to Corollary 14. We denote by $W$ the subspace that they span.

The conclusion of Corollary 17 is clear in the special case where $\left(u_{1}, \ldots, u_{k}\right)=\left(w_{1}, \ldots, w_{k}\right)$. Indeed, we can take $U^{\prime}$ to be the space spanned by the vectors $w_{j}^{\prime}=\left(e_{2 j-1}-i e_{2 j}\right) / 2(1 \leq j \leq r)$. We will reduce the general case to this one thanks to Witt's extension theorem.

Consider then a linear map $\phi: W \rightarrow U \oplus V$ such that $\phi\left(w_{j}\right)=c_{j} u_{j}$ for $j \leq k$. Choose nonzero constants $c_{j}$ so that $\left\langle\phi\left(w_{j}\right), \phi\left(w_{j}\right)\right\rangle=\left\langle w_{j}, w_{j}\right\rangle$ for all $j$ (we may, and will, take $c_{j}=1$ for $j \leq r$ ). This map is designed to preserve the inner product on $W$, and it is injective since the $u_{j}$ are linearly independent. It can thefore be extended to an isometry $F$ of $\mathbb{C}^{n}$ by Theorem 15. Then we take $U^{\prime}$ to be the space spanned by the vectors $u_{j}^{\prime}=F\left(w_{j}^{\prime}\right)$. 


\section{Orthogonal Waring decomposition}

Orthogonal Waring decomposition has been studied in particular in 24, 5] where it is called orthogonal decomposition of symmetric tensors. Theorem 20 below provides an alternative and elementary treatment for the case of order 3 tensors. Orthogonal decompositions are defined in [24, 5] in the language of tensors rather than in the language of polynomials used in Definition 1. The two definitions are indeed equivalent:

Proposition 18. Let $f \in \mathbb{R}\left[x_{1}, \ldots, x_{n}\right]_{d}$ be a homogeneous polynomial of degree $d$ and let $S$ be the corresponding symmetric tensor of order $d$. The two following properties are equivalent:

(i) $f$ admits an orthogonal Waring decomposition.

(ii) $S$ is "symmetrically odeco" [5], i.e., can be written as

$$
\sum_{i=1}^{k} \pm v_{i}^{\otimes d}
$$

where $v_{1}, \ldots, v_{k}$ are nonzero, pairwise orthogonal vectors in $\mathbb{R}^{n}$.

Proof. Suppose that $f(x)=g(A x)$ where $A$ is an orthogonal matrix and $g$ is as in (11). By definition, $f(x)=\sum_{i=1}^{n} a_{i}\left\langle v_{i}, x\right\rangle^{d}$ where the $v_{i}$ are the rows of $A$. Therefore we have $S=\sum_{i=1}^{n} a_{i} v_{i}^{\otimes d}$. We obtain a decomposition of the form (ii) by dropping the terms with $a_{i}=0$, and dividing the remaining $v_{i}$ by $\left|a_{i}\right|^{1 / d}$.

Conversely, if $S$ is symmetrically odeco we can extend $v_{1}, \ldots, v_{k}$ to an orthogonal basis $v_{1}, \ldots, v_{n}$ of $\mathbb{R}^{n}$ and we can normalize these vectors to obtain an orthonormal basis $w_{1}, \ldots, w_{n}$. This yields a decomposition of the form $S=\sum_{i=1}^{n} a_{i} w_{i}^{\otimes d}$, and $f$ admits the orthogonal Waring decomposition $f(x)=\sum_{i=1}^{n} a_{i}\left\langle w_{i}, x\right\rangle^{d}$.

Note that for odd $d$, the \pm signs can be dropped from (ii).

The above equivalence is very straightforward but we point out that it fails over the field of complex numbers, namely, it is no longer the case that (ii) implies (i). This is due to the fact that some vectors $v_{i} \in \mathbb{C}^{n}$ could be isotropic (in the sense of Definition 12) and such vectors cannot be normalized: consider for instance the polynomial $f=\left(x_{1}+i x_{2}\right)^{3}$ of Example 29 and the corresponding tensor $S=(1, i)^{\otimes 3}$. As a result, over $\mathbb{C}$ we no longer have a single notion of "symmetric orthogonal decomposition." In Section 3.2 we propose a natural version of (ii) for the field of complex numbers, which we denote $\operatorname{SODECO}_{n}(\mathbb{C})$. 6 We investigate the relationship of this class of tensors with $\mathrm{OW}_{n}(\mathbb{C})$ in Section 3.2 and with $\overline{\mathrm{OW}_{n}(\mathbb{C})}$ in Section 3.3. As one of our main results we will show that:

\footnotetext{
${ }^{6}$ The notation SODECO stands for "symetrically odeco."
} 
Theorem 19. For every $n \geq 1$ we have

$$
\mathrm{OW}_{n}(\mathbb{C}) \subseteq \operatorname{SODECO}_{n}(\mathbb{C}) \subseteq \overline{\mathrm{OW}_{n}(\mathbb{C})}
$$

These two inclusions are strict for every $n \geq 2$.

As we will see in Section 4, the situation for ordinary tensors is similar: we have a single notion of "orthogonal tensor decomposition" over $\mathbb{R}$ but not over $\mathbb{C}$.

\subsection{Orthogonal Waring decomposition over the reals}

Theorem 20. A real symmetric tensor of order 3 admits an orthogonal Waring decomposition if and only if its slices pairwise commute. In particular, the set of symmetric tensors of order 3 and size $n$ that admit an orthogonal Waring decomposition is the zero set of a system of $n^{2}(n-1)^{2} / 4$ polynomial equations of degree 2 in $\left(\begin{array}{c}n+2 \\ 3\end{array}\right)$ variables.

Proof. Let $f \in \mathbb{R}\left[x_{1}, \ldots, x_{n}\right]_{3}$ be the degree 3 form associated to the symmetric tensor, and let $S_{1}, \ldots, S_{n}$ be the slices. We first consider the case where $f$ admits the orthogonal Waring decomposition $f(x)=g(A x)$. Recall from (44) that the slices satisfy $S_{k}=A^{T} D_{k} A$ where the matrices $D_{k}$ are diagonal. Since $A^{T}=A^{-1}$ the slices are simultaneously diagonalizable and they must therefore commute.

For the converse, assume now that the slices commute. Recall that the matrices $A_{1}, \ldots, A_{n}$ in (6) satisfy $A_{k}=6 S_{k}$. It is a well know fact of linear algebra that a set of matrices is simultaneously diagonalizable iff these matrices commute and each matrix is diagonalizable ([12], Theorem 1.3.21). The latter assumption is satisfied since the $A_{i}$ are real symmetric matrices. By Theorem [11 there are diagonal matrices $D_{1}, \ldots, D_{n}$ and an orthogonal matrix $A$ such that $A_{i}=A^{T} D_{i} A$. Therefore $H_{f}(x)=x_{1} A_{1}+\cdots+x_{n} A_{n}=A^{T} D(x) A$ where $D(x)$ is a diagonal matrix whose entries are linear forms in $x_{1}, \ldots, x_{n}$. Consider now the polynomial $g(x)=f\left(A^{-1} x\right)=f\left(A^{T} x\right)$. By Fact 5 we have

$$
H_{g}(x)=A H_{f}(A x) A^{T}=D(A x)
$$

and $H_{g}$ is a diagonal matrix. By Lemma 5 is of form (10) and $f(x)=g(A x)$ admits an orthogonal Waring decomposition.

The resulting polynomial system contains $n^{2}(n-1)^{2} / 4$ equations because we have to express the commutativity of $n(n-1) / 2$ pairs of matrices. Each commutativity condition yields $n(n-1) / 2$ equations (Indeed, two symmetric matrices commute iff their product is symmetric as well; it therefore suffices to express the equality of each upper triangular entry of the product with the corresponding lower triangular entry). 
In the remainder of Section 3.1 we elucidate the connection between the linear algebraic approach leading to Theorem 20 and the approach from [5]. Given a 3-dimensional symmetric tensor $T$, the authors of that paper define on $V=\mathbb{R}^{n}$ a certain bilinear map $V \times V \rightarrow V,(u, v) \mapsto u$.v. This map is defined on elements of the standard basis $e_{1}, \ldots, e_{n}$ of $\mathbb{R}^{n}$ by:

$$
e_{i} . e_{j}=\sum_{l=1}^{n} T_{i j l} e_{l} \text {. }
$$

One can then extend this map to the whole of $V \times V$ by bilinearity (in [5] they actually give an equivalent coordinate free definition of this map). Then they establish a connection between the associativity of this map and symmetric odeco decompostions:

Theorem 21. The tensor $T$ is symmetrically odeco if and only if $(V,$.$) is$ associative.

In light of Theorem 20 and Proposition 49, we can conclude from Theorem 21 that $(V,$.$) is associative if and only if the slices of T$ commute. We now give an alternative proof of this equivalence by a direct calculation. In fact, we show that this equivalence holds for an arbitrary field (note indeed that (8) makes sense for an arbitrary field and not just for the field of real numbers).

Theorem 22. Let $T$ be a symmetric tensor of order 3 and size $n$, with entries in an arbitrary field $K$. The slices of $T$ commute if and only if $\left(K^{n},.\right)$ is associative.

Proof. Assume first that $\left(K^{n},.\right)$ is associative. In particular, for vectors of the standard basis we have $\left(e_{i} \cdot e_{j}\right) \cdot e_{k}=e_{i} \cdot\left(e_{j} \cdot e_{k}\right)$. By (8), the left-hand side is equal to:

$$
\sum_{l} T_{i j l}\left(e_{l} \cdot e_{k}\right)=\sum_{l} T_{i j l}\left(\sum_{m} T_{l k m} e_{m}\right)=\sum_{m}\left(\sum_{l} T_{i j l} T_{l k m}\right) e_{m} .
$$

A similar computation shows that

$$
e_{i} \cdot\left(e_{j} . e_{k}\right)=\sum_{m}\left(\sum_{l} T_{j k l} T_{i l m}\right) e_{m} .
$$

Therefore we have

$$
\sum_{l} T_{i j l} T_{l k m}=\sum_{l} T_{j k l} T_{i l m}
$$

for every $m$. By symmetry of $T$, the left hand side is equal to $\left(T_{i} T_{k}\right)_{j m}$ where $T_{1}, \ldots, T_{n}$ denote the slices of $T$. As to the right-hand side, it is equal to $\left(T_{k} T_{i}\right)_{j m}$. Hence we have shown that $T_{i} T_{k}=T_{k} T_{i}$ for any $i, k$.

Conversely, assume now that the slices $T_{1}, \ldots, T_{n}$ commute. The above computation shows that $\left(e_{i} \cdot e_{j}\right) \cdot e_{k}=e_{i} \cdot\left(e_{j} \cdot e_{k}\right)$, i.e., associativity holds for basis vectors. The associativity of $\left(K^{n},.\right)$ then follows from bilinearity. 


\subsection{Orthogonal Waring decomposition over the complex numbers}

Theorem 20 does not carry over directly to the field of complex numbers because complex symmetric matrices are not always diagonalizable: consider for instance the matrices $A=\left(\begin{array}{cc}2 i & 1 \\ 1 & 0\end{array}\right)$ or $B=\left(\begin{array}{cc}1 & i \\ i & -1\end{array}\right)$. The second matrix is not diagonalizable since $B^{2}=0$ but $B \neq 0$. For the first one we have $(A-i \mathrm{Id})^{2}=0$ but $A \neq i \mathrm{Id}$.

Theorem 23. A complex symmetric tensor of order 3 admits an orthogonal Waring decomposition if and only if its slices are diagonalizable and pairwise commute.

Proof. Again we consider first the case where the symmetric tensor admits an orthogonal Waring decomposition. The only difference with the real case is that we do need to show that the slices are diagonalizable since this property does not hold true for all complex symmetric matrices. For the slices, this property follows from (44).

For the converse we note that since the slices commute and are now assumed to be diagonalizable, they are simultaneously diagonalizable as in the real case. We can therefore apply the complex case of Theorem 11 and conclude as in the proof of Theorem 20.

Recall that we denote by $\mathrm{OW}_{n}(\mathbb{C})$ the set of polynomials of $\mathbb{C}\left[x_{1}, \ldots, x_{n}\right]_{3}$ that admit an orthogonal Waring decomposition. As explained in Section 2.2, Theorem 23 gives a description of $\mathrm{OW}_{n}(\mathbb{C})$ as a constructible subset of $\mathbb{C}\left[x_{1}, \ldots, x_{n}\right]_{3}$ (i.e. as a subset defined by a Boolean combination of polynomial equalities).

We propose the following adaptation of the notion of a symmetrically odeco tensor ([5] and Proposition [18) from $K=\mathbb{R}$ to $K=\mathbb{C}$.

Definition 24. We denote by $\mathrm{SODECO}_{n}(\mathbb{C})$ the set of symmetric tensors of order 3 that can be written as $\sum_{i=1}^{k} v_{i}^{\otimes 3}$ where $v_{1}, \ldots, v_{k}$ are linearly independent pairwise orthogonal vectors in $\mathbb{C}^{n}$. We use the same notation for the corresponding set of of degree 3 homogenous polynomials in $\mathbb{C}\left[x_{1}, \ldots, x_{n}\right]$.

Over $\mathbb{R}$ the linear independence would follow from the orthogonality of the $v_{i}$ (compare with property (ii) in Proposition 18). We need to add it explicitly in this definition since some of the $v_{i}$ could be isotropic (a situation where pairwise orthogonality does not automatically imply linear independence). Here is a characterization of $\mathrm{OW}_{n}(\mathbb{C})$ in the style of Definition 24:

Proposition 25. $O W_{n}(\mathbb{C})$ is equal to the set of homogeneous polynomials of degree 3 which admit a decomposition of the form

$$
f(x)=\sum_{j=1}^{k}\left\langle u_{j}, x\right\rangle^{3}
$$


for some $k \leq n$, where $u_{1}, \ldots, u_{k}$ are pairwise orthogonal non-isotropic vectors of $\mathbb{C}^{n}$.

Proof. It is very similar to the proof of Proposition 18, and applies to homogeneous polynomials of any degree $d$. Suppose indeed that $f(x)=g(A x)$ where $A$ is an orthogonal matrix and $g$ is as in (11). We saw that $f(x)=$ $\sum_{j=1}^{n} a_{j}\left\langle u_{j}, x\right\rangle^{d}$ where the $u_{j}$ are the rows of $A$. These vectors are indeed pairwise orthogonal and non-isotropic since they are the rows of an orthogonal matrix. We obtain the required decomposition by dropping the terms with $a_{j}=0$, and dividing the remaining $u_{j}$ by a $d$-th root of $a_{j}$.

Conversely, assume that we have a decomposition $f(x)=\sum_{j=1}^{k}\left\langle u_{j}, x\right\rangle^{3}$ where $u_{1}, \ldots, u_{k}$ are pairwise orthogonal non-isotropic vectors of $\mathbb{C}^{n}$. We can normalize these vectors and then extend $u_{1}, \ldots, u_{k}$ to an orthonormal basis of $\mathbb{C}^{n}$ using Witt's extension theorem (Theorem [15] and Corollary [16). This shows that $f(x)=g(A x)$ where $A$ is an orthogonal matrix and $g$ is as in (11).

Corollary 26. We have $\mathrm{OW}_{n}(\mathbb{C}) \subseteq \operatorname{SODECO}_{n}(\mathbb{C})$ for every $n \geq 1$. This inclusion is strict for every $n \geq 2$.

Proof. The inclusion is immediate from Definition 24 and Proposition 25. In order to show that it is strict for $n=2$ consider the symmetric tensor $S=$ $e^{\otimes 3}$ where $e=(1, i)$ (or in an equivalent language, consider the polynomial $\left.f\left(x_{1}, x_{2}\right)=\left(x_{1}+i x_{2}\right)^{3}\right)$. The first slice of $S$ is the matrix $B$ at the beginning of Section 3.2, and it is not diagonalizable. Hence $S \notin \mathrm{OW}_{2}(\mathbb{C})$ by Theorem 23. and it is clear that $S \in \operatorname{SODECO}_{2}(\mathbb{C})$.

This example can be generalized to any $n \geq 2$ by adding "dummy variables" $x_{3}, \ldots, x_{n}$. Namely, if we set $f\left(x_{1}, \ldots, x_{n}\right)=\left(x_{1}+i x_{2}\right)^{3}$, it is still the case that the matrix of the quadratic form $\partial f / \partial x_{1}$ is not diagonalizable. Hence $f \notin \mathrm{OW}_{n}(\mathbb{C})$ by Theorem 23 .

In Section 3.3 we will use the same example to show that $\mathrm{OW}_{n}(\mathbb{C})$ is not closed for $n \geq 2$ (see Example 29 and Remark 30).

\subsection{Closure properties from below}

We will now study the closure $\overline{\mathrm{OW}_{n}(\mathbb{C})}$ of $\mathrm{OW}_{n}(\mathbb{C})$. This study is justified by the fact that, as we will see in Example $29, \mathrm{OW}_{n}(\mathbb{C})$ is not closed. First, we show that $\mathrm{OW}_{n}(\mathbb{C})$ is "large" in the following sense.

Proposition 27. The set of first slices of symmetric tensors in $\mathrm{OW}_{n}(\mathbb{C})$ is dense in the space of symmetric matrices of size $n$.

An equivalent formulation in the langage of polynomials is that the set of partial derivatives $\partial f / \partial x_{1}$ where $f \in \mathrm{OW}_{n}(\mathbb{C})$ is dense in the space of quadratic forms in $n$ variables. Here the variable $x_{1}$ could of course be replaced by any other variable. 
Proof of Proposition 27. By Lemma 9, it is sufficient to show that the set of diagonalizable symmetric matrices is in the closure of the set of first slices of symmetric tensors in $\mathrm{OW}_{n}(\mathbb{C})$. Consider therefore a diagonalizable symmetric matrix $S$ and the corresponding quadratic form $f(x)=x^{T} S x$. As recalled in Section 2.3, there is an orthogonal matrix $A$ such that $A^{T} S A$ is diagonal. But $A^{T} S A$ is the matrix of the quadratic form $g(x)=f(A x)$. Therefore, $f$ is of the form

$$
f(x)=\sum_{i=1}^{n} a_{i}\left\langle u_{i}, x\right\rangle^{2}
$$

where the $u_{i}$ are the rows of the orthogonal matrix $A^{T}$.

We first consider the case where the coefficients $u_{i 1}$ of $x_{1}$ in the linear forms $\left\langle u_{i}, x\right\rangle$ are all nonzero. In this first case, $S$ can be obtained exactly as the first slice of a symmetric tensor in $\mathrm{OW}_{n}(\mathbb{C})$. This is easily seen by integrating (9) with respect to $x_{1}$ to obtain:

$$
F(x)=\sum_{i=1}^{n} \frac{a_{i}}{3 u_{i 1}}\left\langle u_{i}, x\right\rangle^{3} .
$$

This polynomial is in $\mathrm{OW}_{n}(\mathbb{C})$, and $f=\partial F / \partial x_{1}$ by construction.

In the general case, by Lemma 28 below we can approximate the tuple of vectors $\left(u_{1}, \ldots, u_{n}\right)$ by a sequence of tuples $\left(v_{1}(\epsilon), \ldots, v_{n}(\epsilon)\right)$ of pairwise orthogonal unit vectors such that $u_{i}=\lim _{\epsilon \rightarrow 0} v_{i}(\epsilon)$ and $v_{i 1}(\epsilon) \neq 0$ for all $i$. Our analysis of the first case shows that the quadratic forms

$$
f_{\epsilon}(x)=\sum_{i=1}^{n} a_{i}\left\langle v_{i}(\epsilon), x\right\rangle^{2}
$$

can be obtained exactly as partial derivatives $\partial F_{\epsilon} / \partial x_{1}$ with $F_{\epsilon} \in \mathrm{OW}_{n}(\mathbb{C})$. This completes the proof since $f=\lim _{\epsilon \rightarrow 0} f_{\epsilon}$.

Lemma 28. Any complex orthogonal matrix can be approximated to an arbitrary precision by orthogonal matrices that have no vanishing entries in the first column.

Here, "approximated to an arbitrary precision" means that given an orthogonal matrix $A$, we can construct for any small enough $\epsilon$ an orthogonal matrix $A(\epsilon)$ with the required property (having no vanishing entry in its first column) such that $A=\lim _{\epsilon \rightarrow 0} A(\epsilon)$. Or in other words: the set of orthogonal matrices is equal to the closure of the set of orthogonal matrices that have no vanishing entries in their first columns.

Proof. Let $A$ be an orthogonal matrix and let $c_{1}, \ldots, c_{n}$ be its column vectors. Given $\epsilon \neq 0$ we construct the first column $c_{1}(\epsilon)$ of $A(\epsilon)$ by replacing 
any null entry of $c_{1}$ by $\epsilon$, and normalizing the resulting vector. Then we construct the remaining columns $c_{2}(\epsilon), \ldots, c_{n}(\epsilon)$ by applying the Gram-Schmidt orthonormalization process to $c_{1}(\epsilon), c_{2}, \ldots, c_{n}$. For $\epsilon$ small enough, this process will yield pairwise orthogonal vectors $c_{1}(\epsilon), c_{2}(\epsilon), \ldots, c_{n}(\epsilon)$ of unit length $\left(\left\langle c_{j}(\epsilon), c_{j}(\epsilon)\right\rangle=1\right)$ such that $\lim _{\epsilon \rightarrow 0} c_{j}(\epsilon)=c_{j}$ for all $j$. We therefore obtain an orthogonal matrix $A(\epsilon)$ such that $A=\lim _{\epsilon \rightarrow 0} A(\epsilon)$, and by construction the entries of the first column of $A(\epsilon)$ are all nonzero.

In order to properly justify this construction it is important to note that, contrary to the case of real vectors 7 the Gram-Schmidt process cannot be carried out on all lists of linearly independent complex vectors. This is due to the existence of isotropic vectors in $\mathbb{C}^{n}$ (such vectors cannot be normalized). In the present situation this process will nonetheless succeed for any small enough $\epsilon$ due to the property $\lim _{\epsilon \rightarrow 0} c_{j}(\epsilon)=c_{j}$, which can be proved by induction on $j$. Since $\left\langle c_{j}, c_{j}\right\rangle=1$ for all $j$, this property guarantees that we will not attempt to normalize any isotropic vector.

We now give an example showing that $\mathrm{OW}_{2}(\mathbb{C})$ is not closed. It can be easily generalized to any $n \geq 2$ by adding "dummy variables" like in the proof of Proposition 26.

Example 29. The polynomial $f\left(x_{1}, x_{2}\right)=\left(x_{1}+i x_{2}\right)^{3}$ is in $\overline{\mathrm{OW}_{2}(\mathbb{C})} \backslash$ $\mathrm{OW}_{2}(\mathbb{C})$. One can show that $f \notin \mathrm{OW}_{2}(\mathbb{C})$ using Theorem $[23$; this can be traced to the fact that the vector $(1, i)$ is isotropic. Indeed, the Hessian matrices of $\partial f / \partial x_{1}$ (respectively, $\left.\partial f / \partial x_{2}\right)$ are:

$$
6\left(\begin{array}{cc}
1 & i \\
i & -1
\end{array}\right), 6 i\left(\begin{array}{cc}
1 & i \\
i & -1
\end{array}\right)
$$

These two matrices commute but they are not diagonalizable (they are nilpotent but nonzero). To see that $f \in \overline{\mathrm{OW}_{2}(\mathbb{C})}$, consider the family of polynomials

$$
f_{\epsilon}\left(x_{1}, x_{2}\right)=\left[x_{1}+(i+\epsilon) x_{2}\right]^{3}+\epsilon\left[\left(1+\epsilon^{2}\right) x_{1}+(i-\epsilon) x_{2}\right]^{3} .
$$

Note that $f=\lim _{\epsilon \rightarrow 0} f_{\epsilon}$. It therefore remains to show that $f_{\epsilon} \in \mathrm{OW}_{2}(\mathbb{C})$ for all $\epsilon$ sufficiently close to 0 . This follows from the fact that the the vector $(1, i+\epsilon)$ is orthogonal to $\left(1+\epsilon^{2}, i-\epsilon\right)$. Indeed, we have $f_{\epsilon}=g_{\epsilon}\left(A_{\epsilon} x\right)$ where $g_{\epsilon}\left(x_{1}, x_{2}\right)=x_{1}^{3}+\epsilon x_{2}^{3}$ and

$$
A_{\epsilon}=\left(\begin{array}{cc}
1 & i+\epsilon \\
1+\epsilon^{2} & i-\epsilon
\end{array}\right) .
$$

We just pointed out that the rows of $A_{\epsilon}$ are orthogonal, and for $\epsilon$ nonzero but small enough they can be normalized since they are not self-orthogonal.

\footnotetext{
${ }^{7}$ For a reminder on the real or complex Hermitian case, see e.g.[12, Section 0.6.4].
} 
We can therefore write $A_{\epsilon}=D_{\epsilon} U_{\epsilon}$ where $D_{\epsilon}$ is a diagonal matrix and $U_{\epsilon}$ orthogonal. Hence we have

$$
f_{\epsilon}=g_{\epsilon}\left(D_{\epsilon} U_{\epsilon} x\right)=g_{\epsilon}^{\prime}\left(U_{\epsilon}\right)
$$

where $g_{\epsilon}^{\prime}=\alpha_{\epsilon} x_{1}^{3}+\beta_{\epsilon} x_{2}^{3}$ for some appropriate coefficients $\alpha_{\epsilon}, \beta_{\epsilon}$. We conclude that (11) provides as needed an orthogonal decomposition of $f_{\epsilon}$.

Remark 30. For an even simpler family of polynomials in $\mathrm{OW}_{2}(\mathbb{C})$ witnessing the fact that $\left(x_{1}+i x_{2}\right)^{3} \in \overline{\mathrm{OW}_{2}(\mathbb{C})}$, one may replace the coefficient $\epsilon$ of $\left[\left(1+\epsilon^{2}\right) x_{1}+(i-\epsilon) x_{2}\right]^{3}$ in (10) by 0 . We obtain the family of polynomials $f_{\epsilon}^{\prime}\left(x_{1}, x_{2}\right)=\left[x_{1}+(i+\epsilon) x_{2}\right]^{3}$, which also has $\left(x_{1}+i x_{2}\right)^{3}$ as its limit. Moreover, the same argument as in Example 29 shows that $f_{\epsilon}^{\prime} \in \mathrm{OW}_{2}(\mathbb{C})$ for any small enough nonzero $\epsilon$.

Alternatively, one can use Theorem 23 to show that $f_{\epsilon}^{\prime} \in \mathrm{OW}_{2}(\mathbb{C})$. The slices of $f_{\epsilon}^{\prime}$ are

$$
S_{\epsilon, 1}=\left(\begin{array}{cc}
1 & i+\epsilon \\
i+\epsilon & (i+\epsilon)^{2}
\end{array}\right), S_{\epsilon, 2}=(i+\epsilon) S_{\epsilon, 1}
$$

These matrices commute. Moreover, for any small enough nonzero $\epsilon$ the characteristic polynomial $\lambda^{2}-\lambda\left[(I+\epsilon)^{2}+1\right]$ of $S_{\epsilon, 1}$ has distinct roots and the two slices will therefore be diagonalizable.

We will now give an example of a polynomial $g \in \overline{\mathrm{OW}_{2}(\mathbb{C})} \backslash \mathrm{OW}_{2}(\mathbb{C})$ for which this property is more delicate to establish than in the previous example.

Example 31. Let $g\left(x_{1}, x_{2}\right)=x_{2}\left(x_{1}+i x_{2}\right)^{2}$. In order to show that $g \in \overline{\mathrm{OW}_{2}(\mathbb{C})}$, one can check (by a tedious calculation or the help of a computer algebra system) that $g(x)=\lim _{\epsilon \rightarrow 0} g_{\epsilon}\left(A_{\epsilon} x\right)$ where $A_{\epsilon}$ is the matrix of Example 29 and

$$
g_{\epsilon}\left(x_{1}, x_{2}\right)=\frac{\left(1+\epsilon^{2}\right)^{3} x_{1}^{3}-x_{2}^{3}}{6 \epsilon} .
$$

The Hessian matrix of $\partial g / \partial x_{2}$ is equal to $2 M$ where

$$
M=\left(\begin{array}{cc}
1 & 2 i \\
2 i & -3
\end{array}\right)
$$

Since -1 is the only eigenvalue of $M$ but $M \neq-\mathrm{Id}$, this matrix is not diagonalizable. Like in Example 29, Theorem 23 therefore implies that $g \notin \mathrm{OW}_{2}(\mathbb{C})$.

For the polynomial $g$ in the above example, we will now prove a stronger result than $g \notin \mathrm{OW}_{2}(\mathbb{C})$. Indeed, it follows from the next lemma that $g$ cannot be expressed as the sum of cubes of two linear forms. 
Lemma 32. Let $h\left(x_{1}, x_{2}\right)=l_{1}\left(x_{1}, x_{2}\right) l_{2}\left(x_{1}, x_{2}\right)^{2}$ where $l_{1}, l_{2}$ are two linear forms. If there are two linear forms $\ell_{1}, \ell_{2}$ such that

$$
h\left(x_{1}, x_{2}\right)=\ell_{1}\left(x_{1}, x_{2}\right)^{3}+\ell_{2}\left(x_{1}, x_{2}\right)^{3}
$$

then $l_{1}, l_{2}$ cannot be linearly independent.

Proof. Suppose that $l_{1}, l_{2}$ are linearly independent. By performing an invertible change of variables we can reduce to the case $l_{1}=x_{1}, l_{2}=x_{2}$. We must therefore show that one cannot write

$$
x_{1} x_{2}^{2}=\ell_{1}\left(x_{1}, x_{2}\right)^{3}+\ell_{2}\left(x_{1}, x_{2}\right)^{3} .
$$

If such a decomposition exists, the linear forms $\ell_{1}, \ell_{2}$ must be linearly independent since $x_{1} x_{2}^{2}$ is not the cube of a linear form. By Lemma 7, the Hessian determinant of the right hand side must be a constant multiple of $\ell_{1} \ell_{2}$. In particular, it is a squarefree polynomial. But a simple computation shows that the Hessian determinant of $x_{1} x_{2}^{2}$ is equal to $-4 x_{2}^{2}$.

The polynomial of Example 31 can be viewed as a polynomial in $n>2$ variables by introducing $n-2$ dummy variables, i.e., we can set $g\left(x_{1}, x_{2}, x_{3}, \ldots, x_{n}\right)=x_{2}\left(x_{1}+i x_{2}\right)^{2}$. Next we show that introducing these extra variables does not help write $g$ as a sum of cubes of linearly independent linear forms. In fact, like Lemma 32 this result holds for any polynomial of the form $l_{1}\left(x_{1}, x_{2}\right) l_{2}\left(x_{1}, x_{2}\right)^{2}$ where the linear forms $l_{1}, l_{2}$ are linearly independent.

Proposition 33. Suppose that

$$
l_{1}\left(x_{1}, x_{2}\right) l_{2}\left(x_{1}, x_{2}\right)^{2}=\ell_{1}\left(x_{1}, \ldots, x_{n}\right)^{3}+\cdots+\ell_{k}\left(x_{1}, \ldots, x_{n}\right)^{3} .
$$

If the linear forms $l_{1}, l_{2}$ are linearly independent then the linear forms $\ell_{1}, \ldots, \ell_{k}$ cannot be linearly independent.

Proof. By performing an invertible change of variables we can assume without loss of generality that $l_{1}=x_{1}$ and $l_{2}=x_{2}$. Let us therefore suppose by contradiction that

$$
x_{1} x_{2}^{2}=\ell_{1}\left(x_{1}, \ldots, x_{n}\right)^{3}+\cdots+\ell_{k}\left(x_{1}, \ldots, x_{n}\right)^{3}
$$

where the $\ell_{i}$ are linearly independent. We consider first the case $k=n$. By Lemma 32 we must have $n \geq 3$. By Lemma 7 the Hessian determinant of the right-hand side of (12) is not identically zero; but for $n \geq 3$, the Hessian determinant of the left hand side (viewed as a polynomial in $n$ variables) is 0 since $x_{1} x_{2}^{2}$ does not depend on the last $n-2$ variables.

It therefore remains to consider the case $k<n$. Since the $\ell_{i}$ are linearly independent, one can set $n-k$ of the variables $x_{1}, \ldots, x_{n}$ to 0 so that the 
resulting linear forms $\ell_{1}^{\prime}, \ldots, \ell_{k}^{\prime}$ in $k$ variables remain linearly independent. If $x_{1}$ or $x_{2}$ are among the variables that have been set to 0 , we obtain from (12) the identity $\ell_{1}^{\prime 3}+\cdots+\ell_{k}^{\prime 3}=0$. This is impossible since the Hessian determinant of the left hand side is nonzero by Lemma 7 . If $x_{1}, x_{2}$ have not been set to 0 we obtain

$$
x_{1} x_{2}^{2}=\ell_{1}^{\prime 3}+\cdots+\ell_{k}^{\prime 3}
$$

and we are back to the case $k=n$ : as shown earlier in the proof, this identity cannot be satisfied if the $\ell_{i}^{\prime}$ are linearly independent.

In the remainder of Section 3.3 we prove the central result of this section 8

Theorem 34. For every $n \geq 1$ we have

$$
\mathrm{OW}_{n}(\mathbb{C}) \subseteq \operatorname{SODECO}_{n}(\mathbb{C}) \subseteq \overline{\mathrm{OW}_{n}(\mathbb{C})}
$$

These two inclusions are strict for every $n \geq 2$.

For the proof we need some elements of the theory of quadratic forms, see Section 2.4.

Lemma 35. Let $\left(u_{1}, \ldots, u_{k}\right)$ be a tuple of linearly independent pairwise orthogonal vectors of $\mathbb{C}^{n}$. This tuple can be approximated to an arbitrary precision by tuples $\left(v_{1}, \ldots, v_{k}\right)$ of pairwise orthogonal vectors with $\left\langle v_{j}, v_{j}\right\rangle \neq 0$ for all $j=1, \ldots, k$.

Here, "approximated to an arbitrary precision" means that for every $\epsilon>0$ there is a tuple $\left(v_{1}(\epsilon), \ldots, v_{k}(\epsilon)\right)$ of pairwise orthogonal non-isotropic vectors such that $u_{j}=\lim _{\epsilon \rightarrow 0} v_{j}(\epsilon)$ for all $j=1, \ldots, k$. Note that the orthogonality conditions on the $v_{j}$ together with the conditions $\left\langle v_{j}, v_{j}\right\rangle \neq 0$ imply that these vectors are linearly independent.

Proof. Let us assume that the isotropic vectors appear first in $\left(u_{1}, \ldots, u_{n}\right)$, i.e., $u_{1}, \ldots, u_{r}$ are (for some $r \geq 0$ ) the isotropic vectors in this list. We denote by $U$ the subspace spanned by the isotropic vectors, and by $V$ the subspace spanned by $\left(u_{r+1}, \ldots, u_{k}\right)$. By Corollary 17 we can choose for $U$ an isotropic supplement $U^{\prime}$ of $U$ which is orthogonal to $V$. Let $\left(u_{1}^{\prime}, \ldots, u_{r}^{\prime}\right)$ be a basis of $U^{\prime}$ which satisfies the property $\left\langle u_{i}, u_{j}^{\prime}\right\rangle=\delta_{i j}$ from Theorem 13,

Given $\epsilon$ we approximate $u_{j}$ by $v_{j}(\epsilon)=u_{j}+\epsilon u_{j}^{\prime}$ for $j \leq r$. We set $v_{j}(\epsilon)=u_{j}$ for $r+1 \leq j \leq k$. Obviously, we have $u_{j}=\lim _{\epsilon \rightarrow 0} v_{j}(\epsilon)$ for all $j$.

For $j \leq r$ and $l>r$ we have

$$
\left\langle v_{j}(\epsilon), v_{l}(\epsilon)\right\rangle=\left\langle u_{j}+\epsilon u_{j}^{\prime}, u_{l}\right\rangle=0
$$

\footnotetext{
${ }^{8}$ The theorem's statement already appears as Theorem 19 before Section 3.1 We reproduce it here for the reader's convenience.
} 
since $\left\langle u_{j}, u_{l}\right\rangle=0\left(u_{1}, \ldots, u_{k}\right.$ are pairwise orthogonal $)$ and $\left\langle u_{j}^{\prime}, u_{l}\right\rangle=0\left(U^{\prime}\right.$ is orthogonal to $V)$. For $j, l>r$ we have $\left\langle v_{j}(\epsilon), v_{l}(\epsilon)\right\rangle=\left\langle u_{j}, u_{l}\right\rangle$; this is nonzero for $j=l$, and 0 for $j \neq l$. For $j, l \leq r$ we have

$$
\left\langle v_{j}(\epsilon), v_{l}(\epsilon)\right\rangle=\left\langle u_{j}+\epsilon u_{j}^{\prime}, u_{l}+\epsilon u_{l}^{\prime}\right\rangle=\epsilon\left[\left\langle u_{j}, u_{l}^{\prime}\right\rangle+\left\langle u_{j}^{\prime}, u_{l}\right\rangle\right]
$$

since $U$ and $U^{\prime}$ are totally isotropic. This is equal to $2 \epsilon$ for $j=l$, and to 0 for $j \neq l$.

Proof of Theorem 34. The first inclusion, and the fact it is strict for $n \geq 2$, is given by Corollary 26. For the second inclusion, consider a symmetric tensor $S \in \operatorname{SODECO}_{n}(\mathbb{C})$ and the corresponding decomposition $S=\sum_{j=1}^{k} u_{j}^{\otimes 3}$ from Definition 24. Lemma 35 provides us with pairwise orthogonal nonisotropic vectors $v_{j}(\epsilon)$ such that $u_{j}=\lim _{\epsilon \rightarrow 0} v_{j}(\epsilon)$. As a result, $S$ is the limit of the symmetric tensors $S(\epsilon)=\sum_{j=1}^{k} v_{j}(\epsilon)^{\otimes 3}$. These tensors are in $\mathrm{OW}_{n}(\mathbb{C})$ by Proposition 25.

It remains to show that the second inclusion is strict for $n \geq 2$. The witness of this separation will be the polynomial

$$
g_{n}\left(x_{1}, \ldots, x_{n}\right)=x_{2}\left(x_{1}+i x_{2}\right)^{2} .
$$

We have shown in Example 31 that $g_{2} \in \overline{\mathrm{OW}_{2}(\mathbb{C})}$. For $n>2$ the property $g_{n} \in \overline{\mathrm{OW}_{n}(\mathbb{C})}$ continues to hold since $\mathrm{OW}_{2}(\mathbb{C}) \subseteq \mathrm{OW}_{n}(\mathbb{C}) 9$ Finally, Proposition 33 shows that $g_{n} \notin \mathrm{SODECO}_{n}(\mathbb{C})$. Note that the polynomial of Example 29 cannot be used instead of $g_{n}$ to prove this separation since it belongs to $\mathrm{SODECO}_{n}(\mathbb{C})$.

\subsection{The ASD property}

In this section we investigate $\overline{\mathrm{OW}_{n}(\mathbb{C})}$ "from above", i.e., we find properties that must be satisfied by all of its elements. Here is a simple example of such a property:

Proposition 36. If $f \in \overline{\mathrm{OW}_{n}(\mathbb{C})}$ the matrices $A_{1}, \ldots, A_{n}$ in (6) pairwise commute.

Proof. This follows from Theorem 23 and the fact that the commutativity conditions $A_{i} A_{j}-A_{j} A_{i}=0$ are closed.

Are there additional conditions that must be satisfied by the polynomials in $\overline{\mathrm{OW}_{n}(\mathbb{C})}$ besides the above commutativity conditions? The $A S D$ property defined in the introduction turns out to be useful for the investigation of this question. There is a significant body of work on the ASD property, see [22, 21] and the references therein.

\footnotetext{
${ }^{9}$ For this inclusion to make sense we must of course continue to view a polynomial in two variables as a polynomial in $n$ variables.
} 
Proposition 37. If $f \in \overline{\mathrm{OW}_{n}(\mathbb{C})}$ the matrices $A_{1}, \ldots, A_{n}$ in (6) are approximately simultaneously diagonalizable.

Proof. This follows immediately from Theorem 23 and the already mentioned fact that a collection of matrices is simultaneously diagonalizable iff each matrix is diagonalizable and they pairwise commute.

It is not clear whether the converse of this proposition holds because a tuple $\left(B_{1}, \ldots, B_{n}\right)$ approximating $\left(A_{1}, \ldots, A_{n}\right)$ in the sense of Definition 3 might not come from a symmetric tensor. In fact, it is not clear whether a tuple of symmetric matrices satisfying the ASD property can always be approximated in the sense of Definition 3 by a tuple of simultaneously diagonalizable symmetric matrices.

In the next theorem we collect some properties that must be satisfied by any tuple of matrices satisfying the ASD property.

Theorem 38. Any tuple $\left(A_{1}, \ldots, A_{k}\right)$ of approximately simultaneously diagonalizable matrices of $M_{n}(\mathbb{C})$ must satisfy the following properties:

(i) The matrices $A_{1}, \ldots, A_{k}$ pairwise commute.

(ii) The subalgebra of $M_{n}(\mathbb{C})$ generated by $A_{1}, \ldots, A_{k}$ and the identity matrix is of dimension at most $n$.

(iii) The centralizer of $A_{1}, \ldots, A_{k}$ is of dimension at least $n$.

The first property can be found in 22 and follows easily from the fact (already used in the proof of Proposition 36) that the commutativity of two matrices is a closed condition. The second property is established in the same paper, and the third one is Theorem 6.6.2 from [21]. From Theorem 38 and Proposition 37 we have:

Theorem 39. If $f \in \overline{\mathrm{OW}_{n}(\mathbb{C})}$ the matrices $A_{1}, \ldots, A_{n}$ in (6) must satisfy the following properties:

(i) $A_{i} A_{j}=A_{j} A_{i}$ for all $i, j$.

(ii) The subalgebra of $M_{n}(\mathbb{C})$ generated by $A_{1}, \ldots, A_{n}$ and the identity matrix is of dimension at most $n$.

(iii) The centralizer of $A_{1}, \ldots, A_{n}$ is of dimension at least $n$.

In the remainder of this section we show that the converse of this theorem does not hold: the conjunction of these 3 conditions does not imply membership in $\overline{\mathrm{OW}_{n}(\mathbb{C})}$.

Theorem 40. For every $n \geq 21$ there is a polynomial $f \in \mathbb{C}\left[X_{1}, \ldots, X_{n}\right]_{3}$ which satisfies conditions (i), (ii) and (iii) of Theorem 39 but does not belong to $\overline{\mathrm{OW}_{n}(\mathbb{C})}$. 
It is already known that the 3 properties of Theorem 38 together still do not suffice to imply the ASD property [10, 21]. Theorem 40 does not follow from this result since the counterexamples in [10, 21] are not constructed from symmetric tensors.

Before giving the proof of Theorem 40 we need some preliminary results. In particular, we need the following lemma about the Waring rank of forms of degree 3 (recall that the Waring rank of a homogeneous polynomial $f$ of degree $d$ is the smallest $r$ such that $f$ can be written as a sum of $r d$-th powers of linear forms).

Lemma 41. If $v, r$ are two integers with $r \leq\lceil(v+1)(v+2) / 6\rceil$, there exists a degree 3 homogeneous polynomial in $v$ variables which can be written as a sum of $r$ cubes of linear forms, but not as a sum of fewer cubes.

Proof. For $v \geq 2$, let $w(v)$ be the maximum Waring rank of any homogeneous polynomial of degree 3 in $v$ variables. We claim that for every $r \leq w(v)$ there exists a degree 3 homogeneous polynomial in $v$ variables which can be written as a sum of $r$ cubes of linear forms, but not as a sum of fewer cubes. This is easily proved by downward induction on $r$. Indeed, this property holds true for $r=w(v)$ by definition of the maximum rank. For the induction step, assume by contradiction that for some $r<w(v)$ every sum of $r$ cubes can be rewritten as a sum of $r-1$ cubes. Then we could also rewrite every sum of $r+1$ cubes as a sum of $r$ cubes (by rewriting the sum of the first $r$ cubes as a sum of $r-1$ cubes).

Thus it remains to show that $w(v) \geq\lceil(v+1)(v+2) / 6\rceil$. This follows from a simple dimension count. Indeed, $\mathbb{C}\left[x_{1}, \ldots, x_{v}\right]_{3}$ is of dimension $\left(\begin{array}{c}v+2 \\ 3\end{array}\right)$ but the set of polynomials that can be expressed as a sum of $r$ cubes is of dimension 10 at most $r v$ since we have $v$ parameters (the coefficients of the corresponding linear function) for each cube. Hence $w(v) \geq\left(\begin{array}{c}v+2 \\ 3\end{array}\right) / v$.

Remark 42. The above dimension count shows that $\lceil(v+1)(v+2) / 6\rceil$ is a lower bound on the Waring rank of a generic polynomial of $\mathbb{C}\left[x_{1}, \ldots, x_{v}\right]_{3}$. Moreover, it is known (but much harder to prove) that this is the exact value of the generic Waring rank, except for $v=5$ where the generic rank is 8 instead of 7 . This is the degree 3 case of the celebrated Alexander-Hirschowitz theorem [1, 6], which determines the exact value of the generic Waring rank for any number of variables and any degree. The exact value of the maximum Waring rank does not seem to be known in general, but it is never more than twice the generic rank [4].

For the proof of Theorem 40 we also need the notion of border Waring rank.

\footnotetext{
${ }^{10}$ as a constructible subset of $\mathbb{C}\left[x_{1}, \ldots, x_{v}\right]_{3}$
} 
Definition 43. A polynomial $f \in \mathbb{C}\left[X_{1}, \ldots, X_{n}\right]_{3}$ is of border Waring rank at most $r$ if there is a sequence $\left(f_{k}\right)_{k \geq 0}$ of polynomials of $\mathbb{C}\left[X_{1}, \ldots, X_{n}\right]_{3}$ such that $f=\lim _{k \rightarrow+\infty} f_{k}$ and each $f_{k}$ is of Waring rank at most $r$.

In other words, $f$ is of border Waring rank at most $r$ if it is in the closure of the set of polynomials of Waring rank at most $r$.

Lemma 44. For any integer $v$ there exists a polynomial $f \in \mathbb{C}\left[X_{1}, \ldots, X_{v}\right]_{3}$ of border Waring rank at least $\lceil(v+1)(v+2) / 6\rceil$.

Proof. We have seen in the proof of Lemma 41 that if $r<(v+1)(v+2) / 6$, the set of polynomials of Waring rank at most $r$ is of dimension less than $\operatorname{dim} \mathbb{C}\left[X_{1}, \ldots, X_{v}\right]_{3}$. Taking the closure does not increase the dimension, so the closure remains of dimension less than $\operatorname{dim} \mathbb{C}\left[X_{1}, \ldots, X_{v}\right]_{3}$ and there must exist a polynomial in its complement.

The following remark will be useful for the proof of Theorem 40.

Remark 45. For any $n>w$ we can view any polynomial $f \in \mathbb{C}\left[X_{1}, \ldots, X_{w}\right]_{3}$ as a polynomial in $\mathbb{C}\left[X_{1}, \ldots, X_{n}\right]_{3}$ which does not depend on its last $n-w$ variables, and this does not change the border Waring rank of $f$. Let us indeed denote by $r_{w}$ (respectively, $r_{n}$ ) the border Waring rank of $f$ when viewed as a polynomial in $w$ (respectively, $n$ ) variables. By definition of $r_{w}$ there is a sequence $\left(f_{k}\right)_{k \geq 0}$ of polynomials in $\mathbb{C}\left[X_{1}, \ldots, X_{w}\right]_{3}$ of Waring rank at most $r_{w}$ such that $f=\lim _{k \rightarrow+\infty} f_{k}$. The $f_{k}$ can also be viewed as polynomials in $n$ variables, hence $r_{n} \leq r_{w}$. Likewise, there is a sequence $\left(g_{k}\right)_{k \geq 0}$ of polynomials in $\mathbb{C}\left[X_{1}, \ldots, X_{n}\right]_{3}$ of Waring rank at most $r_{n}$ such that $f=$ $\lim _{k \rightarrow+\infty} g_{k}$. The polynomials $f_{k}^{\prime}\left(X_{1}, \ldots, X_{w}\right)=g_{k}\left(X_{1}, \ldots, X_{v}, 0, \ldots, 0\right)$ are of Waring rank at most $r_{n}$, and $f=\lim _{k \rightarrow+\infty} f_{k}^{\prime}$. Hence $r_{w} \leq r_{n}$.

Finally, we need two results involving isotropic vectors.

Proposition 46. If a symmetric tensor $S$ admits a decomposition of the form

$$
S=\sum_{i=1}^{r} u_{i}^{\otimes 3}
$$

where the $u_{i}$ are pairwise orthogonal nonzero vectors then the slices of $S$ commute. Moreover, for such a decomposition the two following properties are equivalent:

(i) All the $u_{i}$ are isotropic.

(ii) The product of any two (possibly equal) slices of $S$ is equal to 0 .

As a result if (i) holds then in any other decomposition $S=\sum_{i=1}^{q} v_{i}^{\otimes 3}$ where the $v_{i}$ are pairwise orthogonal nonzero vectors, all the $v_{i}$ must be isotropic. 
Proof. The $k$-th slice of a rank one symmetric tensor $u^{\otimes 3}$ is the matrix $u_{k} u u^{T}$, where $u_{k}$ denotes the $k$-th component of $u$. For the tensor $S$ the $k$-th slice $S_{k}$ is therefore equal to $\sum_{i=1}^{r} u_{i k} u_{i} u_{i}^{T}$ where $u_{i k}$ denotes the $k$-th component of $u_{i}$. The product of two slices is therefore given by the formula:

$$
S_{k} S_{l}=\sum_{i, j=1}^{r} u_{i k} u_{j l} u_{i} u_{i}^{T} u_{j} u_{j}^{T} .
$$

In this expression the products $u_{i}^{T} u_{j}$ vanish for $i \neq j$ since the $u_{i}$ are pairwise orthogonal. As a result we obtain

$$
S_{k} S_{l}=\sum_{j=1}^{r} u_{j k} u_{j l}\left(u_{j}^{T} u_{j}\right)\left(u_{j} u_{j}^{T}\right)
$$

and this expression is symmetric in $k$ and $l$.

Next we show that (i) and (ii) are equivalent. Assume first that all the $u_{i}$ are isotropic. Then $S_{k} S_{l}=0$ since the coefficients $u_{j}^{T} u_{j}$ in (13) are all equal to 0 . Conversely, assume that some vector $u_{i}$ is not isotropic. Since $u_{i} \neq 0$, at least one component $u_{i k}$ is nonzero. We claim that $u_{i}$ is an eigenvector of $S_{k}^{2}$ associated to a nonzero eigenvalue, thereby showing that $S_{k}^{2} \neq 0$. Indeed, from (13) and from the orthogonality of the $u_{j}$ we have

$$
S_{k}^{2} u_{i}=u_{i k}^{2}\left(u_{i}^{T} u_{i}\right)^{2} u_{i}
$$

and the eigenvalue $u_{i k}^{2}\left(u_{i}^{T} u_{i}\right)^{2}$ is nonzero as promised. One can also obtain (14) from the observation that $u_{i}$ is an eigenvector of $S_{k}$ associated to the eigenvalue $u_{i k}\left(u_{i}^{T} u_{i}\right)$. The last part of the proposition is clear: if (i) holds then the product of any two slices of $S$ must be equal to 0 since (i) implies (ii). From the converse implication (ii) $\Longrightarrow$ (i) applied to the decomposition $S=\sum_{i=1}^{r} v_{i}^{\otimes 3}$ it follows that the $v_{i}$ are isotropic.

Proposition 47. Let $\left(v_{1}, \ldots, v_{s}\right)$ be a basis of a totally isotropic subspace $V$. The $s^{2}$ matrices $v_{i} v_{j}^{T}$ are linearly independent. Moreover, the product of any two matrices of the form $u v^{T}$ with $u, v \in V$ is equal to 0 (as a result, these matrices span a commutative matrix algebra of dimension $s^{2}$ ).

Proof. Recall from Theorem 13 that there are vectors $v_{1}^{\prime}, \ldots, v_{s}^{\prime}$ such that $v_{j}^{T} v_{i}^{\prime}=\delta_{i j}$ for all $i, j$ and assume that $\sum_{i, j} \alpha_{i, j} v_{i} v_{j}^{T}=0$. Multiplying this identity by any of the $v_{k}^{\prime}$ on the right shows that $\sum_{i} \alpha_{i, k} v_{i}=0$. Since the $v_{i}$ are linearly independent, $\alpha_{1, k}, \ldots, \alpha_{s, k}$ must all be equal to 0 . Since this is true for any $k$ we conclude that the $s^{2}$ matrices are indeed linearly independent. Finally, the product of $u v^{T}$ and $u^{\prime} v^{T}$ where $u, u^{\prime}, v, v^{\prime} \in V$ is equal to 0 since $v^{T} u^{\prime}=0$.

Proof of Theorem 40. Let us denote by $S$ the tensor of the polynomial $f$ to be constructed. We will choose $S$ of the form $\sum_{i=1}^{r} u_{i}^{\otimes 3}$ where the $u_{i}$ belong 
to a totally isotropic subspace of $\mathbb{C}^{n}$. By Proposition 46 the slices of such a tensor commute, so condition (i) will be satisfied. Moreover, since the product of any two slices is equal to 0 the algebra in condition (ii) reduces to the linear subspace spanned by the slices of $S$ and the identity matrix. We have seen in the proof of Proposition 46 that the slices of $S$ are given by the formula:

$$
S_{k}=\sum_{i=1}^{r} u_{i k} u_{i} u_{i}^{T} .
$$

The slices of $S$ are therefore linear combinations of the $r$ matrices $u_{i} u_{i}^{T}$, and the coefficients of the linear combinations are the entries of the matrix $\left(u_{i k}\right)_{1 \leq i \leq r, 1 \leq k \leq n}$. This matrix is of rank at most $n / 2$ since the $u_{i}$ lie in a totally isotropic subspace. As a result the slices span a space of dimension at most $n / 2$. Taking the identity matrix into account, we conclude that the algebra is of dimension at most $1+n / 2 \leq n$. Regarding condition (iii), observe that the centralizer of the slices $S_{1}, \ldots, S_{n}$ contains the centralizer of the matrices $u_{i} u_{i}^{T}$. Therefore, if we take the $u_{i}$ in a totally isotropic subspace of dimension $\lfloor n / 2\rfloor$ it follows from Proposition 47 that the centralizer will be of dimension at least $\lfloor n / 2\rfloor^{2}$. Hence condition (iii) will be satisfied for $n \geq 6$.

It remains to choose the tensor $S$ so that $f \notin \overline{O W_{n}(\mathbb{C})}$. Let $v=\lfloor n / 2\rfloor$ and let $g\left(x_{1}, x_{3}, x_{5}, \ldots, x_{2 v-1}\right)$ be a degree 3 form of border Waring rank at least $\bar{r}=\lceil(v+1)(v+2) / 6\rceil$ (the existence of $g$ is guaranteed by Lemma 44). Note that $\bar{r}>n$ if $n \geq 21$. Let

$$
f\left(x_{1}, x_{2}, x_{3}, \ldots, x_{2 v-1}, x_{2 v}\right)=g\left(x_{1}+i x_{2}, x_{3}+i x_{4}, \ldots, x_{2 v-1}+i x_{2 v}\right) .
$$

Note that $f$ and $g$ have same Waring rank and same border Waring rank. In particular, the border Waring rank of $f$ is greater than $n$ for $n \geq 21$. This shows that $f \notin \overline{\mathrm{OW}_{n}(\mathbb{C})}$ since an orthogonal Waring decomposition is a particular Waring decomposition of size $n$ (for odd $n$ we apply Remark 45 to $f$ with $w=2 v=n-1)$.

Let $\left(e_{1}, \ldots, e_{2 v}\right)$ be the standard basis of $\mathbb{C}^{2 v}$ and let $V$ be the totally isotropic subspace spanned by $e_{1}+i e_{2}, e_{3}+i e_{4}, \ldots, e_{2 v-1}+i e_{2 v}$. Finally, let $S$ be the tensor of $f$ and let $r$ be the Waring rank of $g$. From the corresponding decomposition of $g$ and from (15) we obtain a decomposition $S=\sum_{i=1}^{r} u_{i}^{\otimes 3}$ where the $u_{i}$ belong to $V$. Since this subspace is totally isotropic, as explained at the beginning of the proof $f$ will satisfy conditions (i), (ii) and (iii).

Remark 48. Dimension arguments play an important role in the proof of Theorem 40, and they are captured by the notion of "border Waring rank." These dimension arguments can be written in a more concise way without appealing explicitly to the notion of (border) rank; see the proof of Theorem 71 in the next section for the case of ordinary tensors. 


\section{Orthogonal decomposition of ordinary tensors}

In this section we consider orthogonal decompositions of ordinary (possibly non symmetric) tensors of order 3. Compared to the symmetric case, the results are similar but their statements and proofs are somewhat more complicated. First, we point out that for $K=\mathbb{R}$ Definition 2 agrees with the definition of an "odeco tensor" from [5]:

Proposition 49. Let $t \in \mathbb{R}\left[x_{1}, \ldots, x_{n}\right]_{3}$ be a trilinear form and let $T$ be the corresponding tensor. The two following properties are equivalent:

(i) $t$ admits an orthogonal decomposition.

(ii) $T$ is odeco [5], i.e., can be written as

$$
\sum_{i=1}^{k} u_{i} \otimes v_{i} \otimes w_{i}
$$

where each of the the 3 lists $\left(u_{1}, \ldots, u_{k}\right),\left(v_{1}, \ldots, v_{k}\right),\left(w_{1}, \ldots, w_{k}\right)$ is made of $k$ nonzero, pairwise orthogonal vectors in $\mathbb{R}^{n}$.

This proposition could be extended to multilinear forms of degree $d$ and ordinary tensors of order $d$. We skip the proof, which is essentially the same as for Proposition 18, Like in the symmetric case the equivalence of (i) and (ii) fails over the field of complex numbers due to the existence of isotropic vectors, see Example 61 in Section 4.3. Over $\mathbb{C}$ we will therefore have again two different notions of "orthogonal decomposition." The corresponding classes of tensors are denoted $\mathrm{OT}_{n}(\mathbb{C})$ and $\mathrm{ODECO}_{n}(\mathbb{C})$ (the latter defined in Section 4.3). These are the analogues of the classes $\mathrm{OW}_{n}(\mathbb{C})$ and $\operatorname{SODECO}_{n}(\mathbb{C})$ studied in Section 3 ,

Proposition 50. Let $f(x, y, z)=g(A x, B y, C z)$ where $g$ is a trilinear form with slices $\frac{\partial g}{\partial z_{i}}=x^{T} S_{i} y$. The slices of $f$ are given by the formula:

$$
\frac{\partial f}{\partial z_{k}}=x^{T} T_{k} y
$$

where $T_{k}=A^{T} D_{k} B, D_{k}=\sum_{i=1}^{n} c_{i k} S_{i}$ and the $c_{i k}$ are the entries of $C$.

In particular, if $g$ is as in (2) we have $D_{k}=\operatorname{diag}\left(\alpha_{1} c_{1 k}, \ldots, \alpha_{n} c_{n k}\right)$.

Proof. Differentiating the expression $f(x, y, z)=g(A x, B y, C z)$ shows that

$$
\frac{\partial f}{\partial z_{k}}=\sum_{i=1}^{n} c_{i k} \frac{\partial g}{\partial z_{i}}(A x, B y, C z),
$$

and the result follows by plugging the expression $\frac{\partial g}{\partial z_{i}}=x^{T} S_{i} y$ into this formula.

In the case where $g$ is as in (2), $S_{i}$ is the diagonal matrix with an entry equal to $\alpha_{i}$ at row $i$ and column $i$, and zeroes elsewhere. As a result $D_{k}=$ $\operatorname{diag}\left(\alpha_{1} c_{1 k}, \ldots, \alpha_{n} c_{n k}\right)$. 
Note that the above proof applies to arbitrary matrices $A, B, C$ (orthogonality is not used).

Corollary 51. If a tensor $T \in K^{n \times n \times n}$ admits an orthogonal decompostion, its $n$ slices $T_{1}, \ldots, T_{n}$ satisfy the following conditions: for $1 \leq k, l \leq n$, the matrices $T_{k} T_{l}^{T}$ (respectively, $T_{k}^{T} T_{l}$ ) are symmetric and pairwise commute.

Proof. Let us prove this for the $z$-slices (the same property of course holds for slices in the $x$ and $y$ directions) and for the matrices $T_{k} T_{l}^{T}$.

By Proposition 50, $T_{k}=A^{T} D_{k} B$ where $D_{k}$ is diagonal. We have:

$$
T_{k} T_{l}^{T}=\left(A^{T} D_{k} B\right)\left(B^{T} D_{l} A\right)=A^{T} D_{k} D_{l} A
$$

and this is equal to $T_{l} T_{k}^{T}$ since diagonal matrices commute. We have shown that $T_{k} T_{l}^{T}$ is symmetric. The above equality also implies that these matrices commute since they are simultaneously diagonalizable (recall that $A^{T}=$ $A^{-1}$ ). The proof for the matrices $T_{k}^{T} T_{l}$ is similar, with the roles of $A$ and $B$ exchanged.

Remark 52. The proof for $z$-slices uses only the orthogonality of $A$ and $B$ ( $C$ may be an arbitrary matrix). It relies on the fact that the slices are simultaneously orthogonally equivalent to diagonal matrices.

In the following we will not use explicitly the fact that the matrices in Corollary 51 commute, but we will use the symmetry propery.

\subsection{Orthogonal decomposition of real tensors}

Theorem 53 (simultaneous SVD). Let $T_{1}, \ldots, T_{s}$ be real matrices of size $n$. The following properties are equivalent:

(i) The matrices $T_{k} T_{l}^{T}$ and $T_{k}^{T} T_{l}$ are symmetric for all $k, l \in\{1, \ldots, s\}$.

(ii) The $T_{k}$ are simultaneously orthogonally equivalent to diagonal matrices, i.e., there exist real orthogonal matrices $U$ and $V$ such that the matrices $U^{T} T_{k} V$ are all diagonal.

We have seen in the proof of Corollary 51 that (ii) implies (i). As to the converse, for $k=1$ the matrix $T_{1}$ is an arbitrary real matrix and the required decomposition is the singular value decomposition of $T_{1}$. For $k=2$ this is exercise 2.6.P4 in [12], and the general case is Corollary 9 in [18]. This theorem implies in particular that if the matrices $T_{k} T_{l}^{T}, T_{k}^{T} T_{l}$ are all symmetric then the matrices $T_{k} T_{l}^{T}$ must commute (and the $T_{k}^{T} T_{l}$ commute as well).

Proposition 54. Let $T$ be a real tensor of order 3. The corresponding trilinear form $f(x, y, z)$ admits a decomposition of the form $f(x, y, z)=$ $g(A x, B y, C z)$ with $A, B$ orthogonal and $g$ as in (2) iff the $z$-slices of $T$ satisfy the conditions of Theorem [53. (i): $T_{k} T_{l}^{T}$ and $T_{k}^{T} T_{l}$ are symmetric for all $k, l \in\{1, \ldots, n\}$. 
Proof. If $f(x, y, z)=g(A x, B y, C z)$ with $A, B$ orthogonal and $g$ as in (2), we have already seen (see Remark 52) that the matrices $T_{k} T_{l}^{T}$ and $T_{k}^{T} T_{l}$ are symmetric.

Conversely, if these matrices are symmetric then by Theorem 53 there are real orthogonal matrices $U, V$ such that the matrices $U^{T} T_{k} V$ are all diagonal. Let $h(x, y, z)=f(U x, V y, z)$. By Proposition 50 the $z$-slices of $h$ are diagonal, i.e., we have

$$
\frac{\partial h}{\partial z_{k}}=x^{T} D_{k} y
$$

where $D_{k}$ is a diagonal matrix. This implies that the trilinear form $h$ can be written as

$$
h(x, y, z)=\sum_{i=1}^{n} x_{i} y_{i} c_{i}(z)
$$

where the $c_{i}$ are linear forms (indeed, the presence of any cross-product $x_{i} y_{j} z_{k}$ with $i \neq j$ in $h$ would give rise to a non-diagonal entry in $\left.D_{k}\right)$. We have shown that that $h(x, y, z)=g(x, y, C z)$ where $g(x, y, z)=\sum_{i=1}^{n} x_{i} y_{i} z_{i}$ and $C$ is the matrix with the $c_{i k}$ as entries. As a result we have $f(x, y, z)=$ $h\left(U^{T} x, V^{T} y, z\right)=g\left(U^{T} x, V^{T} y, C z\right)$.

The next result is the main result of Section 4.1. In particular, we recover the result from [5] that the set of real tensors admitting an orthogonal decomposition can be defined by equations of degree 2 .

Theorem 55. For a real tensor $T$ of order 3, the following properties are equivalent:

(i) T admits an orthogonal decomposition.

(ii) The $x, y$ and $z$ slices of $T$ satisfy the conditions of Theorem 53. (i).

For instance, if we denote by $Z_{1}, \ldots, Z_{n}$ the $z$-slices of $T$, the matrices $Z_{k} Z_{l}^{T}$ and $Z_{k}^{T} Z_{l}$ must all be symmetric (and likewise for the $x$ and $y$ slices). Toward the proof of this theorem we need the following lemma.

Lemma 56. Let $f(x, y, z)$ be a real trilinear form and let $A, B, C$ be orthogonal matrices. If the $x, y$ and $z$ slices of $f$ satisfy the conditions of Theorem 53. (i) then the same is true of the form $h(x, y, z)=f(A x, B y, C z)$.

Proof. We can obtain obtain $h$ from $f$ in 3 steps: first perform the linear transformation on the $x$ variables, then on the $y$ variables and finally on the $z$ variables. It is therefore sufficient to prove the lemma for $h(x, y, z)=$ $f(A x, y, z)$. Let $Z_{1}, \ldots, Z_{n}$ be the $z$-slices of $f$ and $Z_{1}^{\prime}, \ldots, Z_{n}^{\prime}$ those of $h$. By Proposition 50 we have $Z_{k}^{\prime}=A^{T} Z_{k}$ so that $Z_{k}^{\prime} Z_{l}^{T}=A^{T}\left(Z_{k} Z_{l}^{T}\right) A$. This matrix is symmetric since $Z_{k} Z_{l}^{T}$ is symmetric, and the same is true of $Z_{k}^{\prime T} Z_{l}^{\prime}=Z_{k}^{T} A A^{T} Z_{l}=Z_{k}^{T} Z_{l}$. The $z$-slices of $h$ therefore satisfy the hypotheses of Theorem 53. (i), and a similar argument applies to the $y$-slices. 
Finally, the $x$-slices of $h$ are $X_{k}^{\prime}=\sum_{i=1}^{n} a_{i k} X_{i}$ where $X_{1}, \ldots, X_{n}$ are the $x$-slices of $f$. As a result,

$$
X_{k}^{\prime} X_{l}^{\prime T}=\sum_{i, j=1}^{n} a_{i k} a_{j l} X_{i} X_{j}^{T} .
$$

This matrix is symmetric since the matrices $X_{i} X_{j}^{T}$ are symmetric by hypothesis. A similar computation shows that $X_{k}^{\prime T} X_{l}^{\prime}$ is symmetric as well. We have therefore shown that all slices of $h$ satisfy the hypotheses of Theorem 53. (i).

Proof of Theorem 55. If $T$ admits an orthogonal decomposition, Proposition 54 shows that the $x, y$ and $z$ slices satisfy the conditions of Theorem53(i). For the converse we begin with a special case: let us assume that the trilinear form $f$ associated to $T$ has the same form as $h$ in (16), i.e.,

$$
f(x, y, z)=\sum_{i=1}^{n} x_{i} y_{i} c_{i}(z)
$$

where the $c_{i}$ are linear forms. Differentiating this expression shows that

$$
\frac{\partial f}{\partial x_{k}}=y_{k} c_{k}(z)
$$

and the $x$-slices of $f$ are therefore the rank-one matrices $X_{k}=D_{k} C$ where $C$ is the matrix with the $c_{i j}$ as entries, and $D_{k}$ is the diagonal matrix with an entry equal to 1 at row $k$ and column $k$, and zeroes elsewhere. By hypothesis the matrix $X_{k} X_{l}^{T}=D_{k} C C^{T} D_{l}$ must be symmetric. But this is a matrix with at most one nonzero entry, located at row $k$ and column $l$. We conclude that $D_{k} C C^{T} D_{l}=0$ for $k \neq l$, i.e., any two distinct rows of $C$ are orthogonal. Normalizing the rows of $C$, we can write

$$
f(x, y, z)=\sum_{i=1}^{n} \alpha_{i} x_{i} y_{i} c_{i}^{\prime}(z)
$$

where $C^{\prime}$ is an orthogonal matrix. Note that the rows of $C$ that are identically zero require a special treatment since they cannot be normalized. If there is one such row we replace it in $C^{\prime}$ by a unit vector that is orthogonal to the $n-1$ other rows of $C$ (there are 2 choices) and the corresponding coefficient $\alpha_{i}$ is set to 0 . More generally, if there are several null rows we pick an orthonormal basis of the orthogonal complement of the span of the rows of $C$, and all the corresponding coefficient $\alpha_{i}$ are set to 0 .

We have therefore shown that $f(x, y, z)=g\left(x, y, C^{\prime} z\right)$ where $g$ is as in (2), i.e., we have obtained an orthogonal decomposition of $f$.

The last step of the proof is a reduction from the general case to (17). Let $T$ be any tensor satisfying property (ii) in the statement of the Theorem, 
and let $f$ be the corresponding trilinear form. We have seen in the proof of Proposition 54 that there are two orthogonal matrices $U, V$ such that the form $h(x, y, z)=f(U x, V y, z)$ is as in (16). By Lemma 56, $h$ also satisfies the hypotheses of Theorem [55] and we have therefore shown in the previous step of the proof that $h$ admits an orthogonal decomposition. The same is true of $f$ since $f(x, y, z)=h\left(U^{T} x, V^{T} y, z\right)$.

\subsection{Orthogonal decomposition of complex tensors}

The following result of Choudhury and Horn [8] is an analogue of Theorem 53 for complex orthogonal equivalence. We state it for square matrices because that is sufficient for our purposes, but it can be generalized to rectangular matrices (see [8] for details).

Theorem 57 (simultaneous diagonalization by complex orthogonal equivalence). Let $T_{1}, \ldots, T_{s}$ be complex matrices of size $n$. The $T_{k}$ are simultaneously orthogonally equivalent to diagonal matrices (i.e., there exist complex orthogonal matrices $U$ and $V$ such that the matrices $U^{T} T_{k} V$ are all diagonal) if and only if the following conditions are satisfied:

(i) For each $k, T_{k}^{T} T_{k}$ is diagonalizable and $\operatorname{rank} T_{k}=\operatorname{rank} T_{k}^{T} T_{k}$.

(ii) The matrices $T_{k} T_{l}^{T}$ and $T_{k}^{T} T_{l}$ are symmetric for all $k, l \in\{1, \ldots, s\}$.

Condition (i) is the necessary and sufficient condition for each $T_{k}$ to be (individually) orthogonally equivalent to a diagonal matrix (8], Theorem 2). It does not appear in Theorem 53 because this condition is automatically satisfied by real matrices. When condition (i) holds, it is shown in Theorem 9 of [8] that condition (ii) is necessary and sufficient for the $T_{k}$ to be simultaneously orthogonally equivalent to diagonal matrices.

We continue with an analogue of Proposition 54.

Proposition 58. Let $T$ be a complex tensor of order 3. The corresponding trilinear form $f(x, y, z)$ admits a decomposition of the form $f(x, y, z)=$ $g(A x, B y, C z)$ with $A, B$ orthogonal and $g$ as in (2) iff the $z$-slices of $T$ satisfy conditions (i) and (ii) of Theorem 57 .

Proof. Assume first that $f(x, y, z)=g(A x, B y, C z)$ with $A, B$ orthogonal and $g$ as in (2). By Proposition 50 the $z$-slices $T_{1}, \ldots, T_{n}$ are of the form $T_{k}=$ $A^{T} D_{k} B$ where $D_{k}$ is diagonal, i.e., the $T_{k}$ are simultaneously orthogonally equivalent to diagonal matrices. They must therefore satisfy the conditions of Theorem 57.

Conversely, assume that the conditions of this theorem are satisfied. Then there are complex orthogonal matrices $U, V$ such that the matrices $U^{T} T_{k} V$ are all diagonal. We can consider the polynomial $h(x, y, z)=$ $f(U x, V y, z)$ and conclude exactly as in the proof of Proposition 54. 
We proceed to the main result of Section 4.2. This is an analogue of Theorem 55 .

Theorem 59. A complex tensor $T$ of order 3 admits an orthogonal decomposition iff the $x, y$ and $z$ slices of $T$ all satisfy conditions (i) and (ii) of Theorem 57, namely, the $x$-slices must satisfy the conditions:

(i) for each $k, X_{k}^{T} X_{k}$ is diagonalizable and $\operatorname{rank} X_{k}=\operatorname{rank} X_{k}^{T} X_{k}$,

(ii) the matrices $X_{k} X_{l}^{T}$ and $X_{k}^{T} X_{l}$ are symmetric for all $k, l \in\{1, \ldots, n\}$, and likewise for the $y$ and $z$ slices.

For the proof we naturally need an analogue of Lemma 56

Lemma 60. Let $f(x, y, z)$ be a complex trilinear form and let $A, B, C$ be orthogonal matrices. If the $x, y$ and $z$ slices of $f$ satisfy conditions (i) and (ii) of Theorem 57 then the same is true of the form $h(x, y, z)=f(A x, B y, C z)$.

Proof. Since the slices of $f$ satisfy condition (ii) of Theorem 57 the same must be true of $h$. Indeed, the proof of Lemma 56 applies verbatim to the present situation. It therefore remains to deal with condition (i). Like in Lemma 56 it suffices to consider the case $h(x, y, z)=f(A x, y, z)$. Let us denote again by $Z_{1}, \ldots, Z_{n}$ the $z$-slices of $f$ and by $Z_{1}^{\prime}, \ldots, Z_{n}^{\prime}$ the $z$-slices of $h$. We saw that $Z_{k}^{\prime}=A^{T} Z_{k}$ by Proposition 50 so that $Z_{k}^{\prime} Z_{k}^{\prime T}=A^{T}\left(Z_{k} Z_{k}^{T}\right) A=$ $A^{-1}\left(Z_{k} Z_{k}^{T}\right) A$. According to condition (i) $Z_{k} Z_{k}^{T}$ is diagonalizable, so the same is true of $Z_{k}^{\prime} Z_{k}^{\prime T}$. Moreover $\operatorname{rank} Z_{k}^{\prime} Z_{k}^{\prime T}=\operatorname{rank} Z_{k} Z_{k}^{T}=\operatorname{rank} Z_{k}=\operatorname{rank} Z_{k}^{\prime}$ and this completes the proof that the $Z_{k}^{\prime}$ satisfy condition (i). A similar argument applies to the $y$-slices.

For the $x$-slices we will use the fact that condition (i) of Theorem 59 is the condition for each slice to be (individually) orthogonally equivalent to a diagonal matrix 11 As pointed out before, this is shown in [8, Theorem 2]. As shown in the proof of Lemma 56, each $x$-slice $X_{k}^{\prime}$ of $h$ is a linear combination of the $x$-slices $X_{1}, \ldots, X_{n}$ of $f$. These slices satisfy condition (i) and (ii) and are therefore simultaneously orthogonally equivalent to diagonal matrices by Theorem 57. Any linear combination of these matrices, and in particular $X_{k}^{\prime}$, is therefore orthogonally equivalent to a diagonal matrix. The $X_{k}^{\prime}$ must therefore satisfy condition (i) by [8, Theorem 2] (note that we only use the "easy" direction of this theorem here).

Proof of Theorem 59. If $T$ admits an orthogonal decomposition, Proposition 58 shows that the $x, y$ and $z$ slices satisfy conditions (i) and (ii) of Theorem 57.

For the converse, we begin as in the proof Theorem [55] with the case where the trilinear form $f$ associated to $T$ is as in (17). This case can be

\footnotetext{
${ }^{11}$ We could also have used this argument for the $y$ and $z$ slices.
} 
treated in the same way except for one important difference. In the proof of Theorem 55 we pointed out that the null rows of $C$ must be treated separately because they cannot be normalized. Over $\mathbb{C}$ there is a further complication: there might be rows $c_{k}$ such that $c_{k}^{T} c_{k}=0$ but $c_{k} \neq 0$; such rows could not be normalized. Fortunately, it turns out that there are no such rows in $C$. Recall indeed from the proof of Theorem [55 that the $k$-th $x$-slice of $T$ is $X_{k}=D_{k} C$, where $D_{k}$ is the diagonal matrix with an entry equal to 1 at row $k$ and column $k$, and zeroes elsewhere. In other words, row $k$ of $X_{k}$ is equal to $c_{k}$ and all other rows are identically 0 . Moreover $X_{k}^{T} X_{k}$ has one entry (at row $k$ and column $k$ ) equal to $c_{k}^{T} c_{k}$ and only 0 's elsewhere. By condition (i) of Theorem 57 we must have $\operatorname{rank} X_{k}=\operatorname{rank} X_{k}^{T} X_{k}$. It follows that $c_{k}^{T} c_{k}=0$ implies $c_{k}=0$. We have therefore shown that all rows of $C$ can be normalized except the null rows. Morever, any two distinct rows are orthogonal as in the proof of Theorem 55. We can therefore conclude essentially as in that proof: the set of normalized non-null rows of $C$ is an orthonormal family, and can therefore be extended to an orthonormal basis of $\mathbb{C}^{n}$ by Witt's theorem (Theorem 15 and Corollary [16). Again, the coefficients $\alpha_{i}$ corresponding to the new vectors in this basis are set to 0 .

It remains to reduce the general case of Theorem 59 to (17) and this can be done essentially as in the proof of Theorem 55. Indeed, let $Z_{1}, \ldots, Z_{n}$ be the $z$-slices of $T$. By Theorem 57 there are orthogonal matrices $U, V$ such that all the matrices $D_{k}=U^{T} Z_{k} V$ are diagonal. We set $h(x, y, z)=$ $f(U x, V y, z)$ like in the proof of Proposition 54 and the $z$-slices of $h$ are the diagonal matrices $D_{k}$ by Proposition 50. This implies that $h$ is as in (16). Moreover, $h$ satisfies conditions (i) and (ii) of Theorem 57 by Lemma 60. This completes the reduction, and the proof of Theorem 59.

\subsection{Closure properties}

In Section 4.2 we gave a characterization of the set $\mathrm{OT}_{n}(\mathbb{C})$ of tensors $T \in$ $\mathbb{C}^{n \times n \times n}$ that admit an orthogonal decomposition. In this section we show that $\mathrm{OT}_{n}(\mathbb{C})$ is not closed, and we find a a nontrivial family of tensors in the closure (Theorem 66). Then we find some of the equations that are satisfied by tensors in $\overline{\mathrm{OT}_{n}(\mathbb{C})}$ (Theorem [69) and we show that these equations do not characterize the closure completely (Theorem 71 ).

First we show that $\mathrm{OT}_{2}(\mathbb{C})$ is not closed by exhibiting a tensor belonging to $\overline{\mathrm{OT}_{2}(\mathbb{C})}$ but not to $\mathrm{OT}_{2}(\mathbb{C})$. This is in fact the same tensor as in Example 29 but we view it as an ordinary tensor instead of a symmetric tensor.

Example 61. Let $f^{1}\left(x_{1}, x_{2}, y_{1}, y_{2}, z_{1}, z_{2}\right)=\left(x_{1}+i x_{2}\right)\left(y_{1}+i y_{2}\right)\left(z_{1}+i z_{2}\right)$. The $z$-slices of $f^{1}$ are:

$$
Z_{1}=\left(\begin{array}{cc}
1 & i \\
i & -1
\end{array}\right), Z_{2}=i\left(\begin{array}{cc}
1 & i \\
i & -1
\end{array}\right)
$$


and the $x$ and $y$ slices are of course the same. We can apply Theorem 59 to show that $f^{1} \notin \mathrm{OT}_{2}(\mathbb{C})$. Indeed, the second part of condition (i) of Theorem 57 is violated: $\operatorname{rank} Z_{1}=1$ but $\operatorname{rank} Z_{1}^{T} Z_{1}=\operatorname{rank} Z_{1}^{2}=0$.

In order to show that $f^{1} \in \overline{\mathrm{OT}_{2}(\mathbb{C})}$, consider the polynomials

$$
g_{\epsilon}=x_{1} y_{1} z_{1}+\epsilon x_{2} y_{2} z_{2}
$$

and $f_{\epsilon}^{1}=g_{\epsilon}\left(A_{\epsilon} x, A_{\epsilon} y, A_{\epsilon} z\right)$ where

$$
A_{\epsilon}=\left(\begin{array}{cc}
1 & i+\epsilon \\
1+\epsilon^{2} & i-\epsilon
\end{array}\right)
$$

is the same matrix as in Example [29. Since $f^{1}=\lim _{\epsilon \rightarrow 0} f_{\epsilon}^{1}$ it remains to show that $f_{\epsilon}^{1} \in \mathrm{OT}_{2}(\mathbb{C})$ for all $\epsilon$ sufficiently close to 0 . We have seen in Example 29 that $A_{\epsilon}=D_{\epsilon} U_{\epsilon}$ where $D_{\epsilon}$ is a diagonal matrix and $U_{\epsilon}$ orthogonal. Hence we have

$$
f_{\epsilon}^{1}=g_{\epsilon}\left(D_{\epsilon} U_{\epsilon} x, D_{\epsilon} U_{\epsilon} y, D_{\epsilon} U_{\epsilon} z\right)=h_{\epsilon}\left(U_{\epsilon} x, U_{\epsilon} y, U_{\epsilon} z\right)
$$

where $h_{\epsilon}=\alpha_{\epsilon} x_{1} y_{1} z_{1}+\beta_{\epsilon} x_{2} y_{2} z_{2}$ for some appropriate coefficients $\alpha_{\epsilon}, \beta_{\epsilon}$. We conclude that (18) provides as needed an orthogonal decomposition of $f_{\epsilon}^{1}$.

We can also give less symmetric examples of polynomials on the boundary of $\mathrm{OT}_{2}(\mathbb{C})$.

Example 62. Let $f^{2}\left(x_{1}, x_{2}, y_{1}, y_{2}, z_{1}, z_{2}\right)=\left(x_{1}+i x_{2}\right)\left(y_{1}+i y_{2}\right) z_{1}$. This polynomial has the same first z-slice as the polynomial $f$ of Example 61; this shows that $f^{2} \notin \mathrm{OT}_{2}(\mathbb{C})$. In order to show that $f^{2} \in \overline{\mathrm{OT}_{2}(\mathbb{C})}$ we can proceed as in the previous example. Indeed we have $f^{2}=\lim _{\epsilon \rightarrow 0} f_{\epsilon}^{2}$ where $f_{\epsilon}^{2}=$ $g_{\epsilon}\left(A_{\epsilon} x, A_{\epsilon} y, z\right)=g_{\epsilon}\left(D_{\epsilon} U_{\epsilon} x, A_{\epsilon} U_{\epsilon} y, z\right)$. From this representation of $f_{\epsilon}^{2}$ we obtain an orthogonal decomposition in the same way as before.

Example 63. Another similar example is:

$$
f^{3}\left(x_{1}, x_{2}, y_{1}, y_{2}, z_{1}, z_{2}\right)=\left(x_{1}+i x_{2}\right) y_{1} z_{1} .
$$

The z-slices of $f^{3}$ are:

$$
Z_{1}=\left(\begin{array}{ll}
1 & 0 \\
i & 0
\end{array}\right), Z_{2}=0
$$

We have $Z_{1}^{T} Z_{1}=0$ and we conclude that our polynomial does not belong to $\mathrm{OT}_{2}(\mathbb{C})$ for the same reason as in Example 61. In order to show that $f^{3} \in \overline{\mathrm{OT}_{2}(\mathbb{C})}$, consider the polynomial $f_{\epsilon}^{3}=g_{\epsilon}\left(A_{\epsilon} x, y, z\right)$ where $g_{\epsilon}$ and $A_{\epsilon}$ are as in the two previous examples. Since $f^{3}=\lim _{\epsilon \rightarrow 0} f_{\epsilon}^{3}$ it remains to show that $f_{\epsilon}^{3} \in \mathrm{OT}_{2}(\mathbb{C})$ for all $\epsilon$ sufficiently close to 0 . 
We have seen that $A_{\epsilon}=D_{\epsilon} U_{\epsilon}$ where $D_{\epsilon}$ is a diagonal matrix and $U_{\epsilon}$ orthogonal. Hence we have

$$
f_{\epsilon}^{3}=g_{\epsilon}\left(D_{\epsilon} U_{\epsilon} x, y, z\right)=g_{\epsilon}^{\prime}\left(U_{\epsilon} x, y, z\right)
$$

where $h_{\epsilon}^{\prime}=\alpha_{\epsilon}^{\prime} x_{1} y_{1} z_{1}+\beta_{\epsilon}^{\prime} x_{2} y_{2} z_{2}$ for some appropriate coefficients $\alpha_{\epsilon}^{\prime}, \beta_{\epsilon}^{\prime}$. We conclude that (19) provides as needed an orthogonal decomposition of $f_{\epsilon}^{3}$.

Like in Section 3.3 we can build on these examples to exhibit more elements of $\overline{\mathrm{OT}_{n}(\mathbb{C})}$.

Definition 64. We denote by $\mathrm{ODECO}_{n}(\mathbb{C})$ the set of tensors of order 3 that can be written as $\sum_{j=1}^{k} u_{j} \otimes v_{j} \otimes w_{j}$ where each of the the 3 lists $\left(u_{1}, \ldots, u_{k}\right)$, $\left(v_{1}, \ldots, v_{k}\right),\left(w_{1}, \ldots, w_{k}\right)$ is made of $k$ linearly independent, pairwise orthogonal vectors in $\mathbb{C}^{n}$. We use the same notation for the corresponding set of trilinear forms in $\mathbb{C}\left[x_{1}, \ldots, x_{n}, y_{1}, \ldots, y_{n}, z_{1}, \ldots, z_{n}\right]$.

The next proposition gives a characterization of $O T_{n}(\mathbb{C})$ in the style of Definition 64, Compare with Proposition 49, where for the real field we did have to introduce explicitly a non-isotropy requirement for the vectors $u_{i}$, $v_{i}, w_{i}$ appearing in the decomposition.

Proposition 65. $O T_{n}(\mathbb{C})$ is equal to the set of order 3 tensors which admit a decomposition of the form $\sum_{j=1}^{k} u_{j} \otimes v_{j} \otimes w_{j}$ for some $k \leq n$, where each of the 3 lists $\left(u_{1}, \ldots, u_{k}\right),\left(v_{1}, \ldots, v_{k}\right),\left(w_{1}, \ldots, w_{k}\right)$ is made of $k$ pairwise orthogonal non-isotropic vectors of $\mathbb{C}^{n}$.

We skip the proof of this proposition because it is entirely parallel to the proof of Proposition 25 for symmetric tensors.

Theorem 66. For every $n \geq 2$ we have $\mathrm{OT}_{n}(\mathbb{C}) \subseteq \mathrm{ODECO}_{n}(\mathbb{C}) \subseteq \overline{\mathrm{OT}_{n}(\mathbb{C})}$. The first inclusion is strict for every $n \geq 2$.

Proof. The first inclusion follows from Definition 64 and Proposition 65. Any one of the 3 examples at the beginning of Section 3.3 shows that the inclusion is strict for $n=2$. Like in the proof of Theorem 34 this can be extended to any $n>2$ by adding dummy variables.

For the second inclusion, consider a tensor $T \in \mathrm{ODECO}_{n}(\mathbb{C})$ and the corresponding decomposition $T=\sum_{j=1}^{k} u_{j} \otimes v_{j} \otimes w_{j}$. We can approximate to an arbitrary precision the tuple $\left(u_{1}, \ldots, u_{k}\right)$ by tuples $\left(u_{1}^{\prime}, \ldots, u_{k}^{\prime}\right)$ satisfying the property of Lemma 35. We can also approximate the tuples $\left(v_{1}, \ldots, v_{k}\right)$ and $\left(w_{1}, \ldots, w_{k}\right)$ by tuples $\left(v_{1}^{\prime}, \ldots, v_{k}^{\prime}\right)$ and $\left(w_{1}^{\prime}, \ldots, w_{k}^{\prime}\right)$ satisfying the same property. In this way we approximate $T$ to an arbitrary precision by the tensors $\sum_{j=1}^{k} u_{j}^{\prime} \otimes v_{j}^{\prime} \otimes w_{j}^{\prime}$, and these tensors belong to $O T_{n}(\mathbb{C})$ by Proposition 65. 
One could show that the second inclusion is strict for every large enough $n$ by adapting the proof of a result about symmetric tensors in an earlier version of this paper: Theorem 30 in [15]. In that theorem the strict inclusion $\operatorname{SODECO}_{n}(\mathbb{C}) \subsetneq \overline{\mathrm{OW}_{n}(\mathbb{C})}$ was obtained for large enough $n$ for a polynomial of "high Waring rank". For Theorem 66 one would start instead from a multilinear polynomial of "high tensor rank" (the details are omitted). It would be interesting to find out whether the second inclusion of Theorem 66 is strict for all $n \geq 2$. Recall that the corresponding result for symmetric tensors was established in Theorem 34 of the present paper.

In order to complete the parallel with the study of symmetric tensors in Section 3 it remains to investigate the closure properties of $\mathrm{OT}_{n}(\mathbb{C})$ "from above". Like in Section 3.4 this will be done thanks to a connection with the ASD property.

Proposition 67. Let $X_{1}, \ldots, X_{n}$ be the $x$-slices of a tensor in $\overline{\mathrm{OT}_{n}(\mathbb{C})}$. The $n^{2}$ matrices $X_{k}^{T} X_{l}(1 \leq k, l \leq n)$ are symmetric and approximately simultaneously diagonalizable (ASD). Likewise, the $n^{2}$ matrices $X_{k} X_{l}^{T}$ are symmetric and ASD. The same properties also hold for the $y$ and $z$-slices.

Proof. Let $X_{1}, \ldots, X_{n}$ be the $x$-slices of a tensor $T \in \mathrm{OT}_{n}(\mathbb{C})$. By Theorem 57 and Theorem 59 there are orthogonal matrices $U$ and $V$ such that the matrices $D_{k}=U^{T} X_{k} V$ are all diagonal (this is actually the easier direction of Theorem (59). Therefore $X_{k}^{T} X_{l}=V D_{k} D_{l} V^{T}$. In particular, these $n^{2}$ matrices are symmetric and simultaneously diagonalizable. Passing to the limit shows that for a tensor in $\overline{\mathrm{OT}_{n}(\mathbb{C})}$, the corresponding $n^{2}$ matrices must be symmetric and ASD. The same argument applies to $X_{k} X_{l}^{T}=U D_{k} D_{l} U^{T}$ and to the $y$ and $z$ slices.

Remark 68. This section deals with ordinary (possibly non symmetric) tensors, but still Proposition 67 shows that we need to use the ASD property for symmetric matrices only. Moreover we only need to consider approximations by simultaneously diagonalizable symmetric matrices since the matrices $V D_{k} D_{l} V^{T}$ in the proof of Proposition 67 are symmetric.

By Proposition 67 and Theorem 38 we have the following analogue of Theorem 39.

Theorem 69. The $x$-slices $X_{1}, \ldots, X_{n}$ of a tensor in $\overline{\mathrm{OT}_{n}(\mathbb{C})}$ must satisfy the following properties:

(i) The matrices $X_{k}^{T} X_{l}$ are symmetric and pairwise commute.

(ii) The subalgebra of $M_{n}(\mathbb{C})$ generated by these $n^{2}$ matrices and by the identity matrix is of dimension at most $n$.

(iii) The centralizer of these matrices is of dimension at least $n$. 
The same properties are satisfied by the matrices $X_{k} X_{l}^{T}$ and by the $y$ and $z$ slices.

Finally, we show that the converse of this theorem does not hold. We will need the following lemma, which follows from Definition 2 and the fact that the complex orthogonal group is of dimension $n(n-1) / 2$.

Lemma 70. $\operatorname{dim} \mathrm{OT}_{n}(\mathbb{C}) \leq 3 n(n-1) / 2+n$.

Theorem 71. For every large enough $n$ there is a tensor of order 3 and size $n$ which satisfies all the properties of Theorem 69 but does not belong to $\overline{\mathrm{OT}_{n}(\mathbb{C})}$.

Proof. As a counterexample we will construct a symmetric tensor $S$, which we are of course free to view as an ordinary tensor. In fact, like in the proof of Theorem 40 we will construct a tensor of the form

$$
S=\sum_{i=1}^{r} u_{i}^{\otimes 3}
$$

where the $u_{i}$ belong to a totally isotropic subspace $V \subseteq \mathbb{C}^{n}$ with $\operatorname{dim} V=$ $\lfloor n / 2\rfloor$. Let us fix such a $V$. Since $r$ is arbitrary, the set $\mathcal{S}$ of tensors of form (20) is a linear space. Its dimension is equal to $\left(\begin{array}{c}v+2 \\ 3\end{array}\right)$ where $v=\lfloor n / 2\rfloor$ (this is the dimension of the space of homogeneous polynomials of degree 3 in $v$ variables).

The $x, y$ and $z$ slices of any $S \in \mathcal{S}$ are the same since $S$ is symmetric. Let us denote them by $S_{1}, \ldots, S_{n}$. We claim that the matrices $S_{k} S_{l}^{T}$ and $S_{k}^{T} S_{l}$ considered in Theorem 69] are all equal to 0 . They will therefore trivially satisfy properties (i), (ii) and (iii). The proof of the claim is simple: by symmetry of $S$ we have $S_{k}^{T}=S_{k}$ and $S_{l}^{T}=S_{l}$, but by Proposition 46 the product of any two slices of $S$ is equal to 0 . Therefore it remains to find an $S \in \mathcal{S}$ which is not in $\overline{\mathrm{OT}_{n}(\mathbb{C})}$. Such an $S$ is guaranteed to exist as soon as $\operatorname{dim} \overline{\mathrm{OT}_{n}(\mathbb{C})}=\operatorname{dim} \mathrm{OT}_{n}(\mathbb{C})<\operatorname{dim} \mathcal{S}$. We have shown that $\operatorname{dim} \mathcal{S}$ is of order $n^{3} / 48$, but $\operatorname{dim} \mathrm{OT}_{n}(\mathbb{C})$ is quadratically bounded by Lemma 70 . Hence we will have $\operatorname{dim} \mathrm{OT}_{n}(\mathbb{C})<\operatorname{dim} \mathcal{S}$ for every large enough $n$ (one can check that it suffices to take $n \geq 68$ ).

In this paper, Theorem 71 is the only result for ordinary tensors with a (slightly) simpler proof than its counterpart for symmetric tensors (Theorem 40). This is due to the fact that we could design the counterexample $S$ in the proof so that $S_{k} S_{l}^{T}=S_{k}^{T} S_{l}=0$.

\section{Acknowledgements}

Nicolas Ressayre made some useful comments on an early version of this paper. I would also like to thank Kevin O'Meara for the encouragements, and Roger Horn 
for sharing his proof of Theorem 11 and its generalizations. The referee's comments led to a strengthening of Theorem 34 and several improvements in the presentation of the paper.

\section{References}

[1] James Alexander and André Hirschowitz. Polynomial interpolation in several variables. Journal of Algebraic Geometry, 4(2):201-222, 1995.

[2] Kim Batselier, Haotian Liu, and Ngai Wong. A constructive algorithm for decomposing a tensor into a finite sum of orthonormal rank-1 terms. SIAM Journal on Matrix Analysis and Applications, 36(3):1315-1337, 2015.

[3] Xiaohui Bei, Shiteng Chen, Ji Guan, Youming Qiao, and Xiaoming Sun. From independent sets and vertex colorings to isotropic spaces and isotropic decompositions. arXiv preprint arXiv:1904.03950, 2019.

[4] Grigoriy Blekherman and Zach Teitler. On maximum, typical and generic ranks. Mathematische Annalen, 362(3-4):1021-1031, 2015.

[5] Ada Boralevi, Jan Draisma, Emil Horobet, and Elina Robeva. Orthogonal and unitary tensor decomposition from an algebraic perspective. Israel Journal of Mathematics, 222(1):223-260, 2017.

[6] Maria Chiara Brambilla and Giorgio Ottaviani. On the Alexander Hirschowitz theorem. Journal of Pure and Applied Algebra, 212(5):1229-1251, 2008.

[7] P. Bürgisser, M. Clausen, and M. A. Shokrollahi. Algebraic Complexity Theory. Springer, 1997.

[8] Dipa Choudhury and Roger A. Horn. An analog of the singular value decomposition for complex orthogonal equivalence. Linear and Multilinear Algebra, 21(2):149-162, 1987.

[9] Pete L. Clark. Quadratic forms Chapter I: Witt's theory. math.uga.edu/pete/quadraticforms.pdf

[10] C De Boor and B Shekhtman. On the pointwise limits of bivariate Lagrange projectors. Linear Algebra and its Applications, 429(1):311-325, 2008.

[11] Roger Horn and Charles Johnson. Topics in matrix analysis. Cambridge University Press, 1994.

[12] Roger Horn and Charles Johnson. Matrix Analysis. Cambridge University Press (second edition), 2013.

[13] Neeraj Kayal. Efficient algorithms for some special cases of the polynomial equivalence problem. In Symposium on Discrete Algorithms (SODA). Society for Industrial and Applied Mathematics, January 2011.

[14] P. Koiran and N. Ressayre. Orbits of monomials and factorization into products of linear forms. arXiv:1807.03663, 2018.

[15] Pascal Koiran. Orthogonal tensor decomposition and orbit closures from a linear algebraic perspective. arXiv:1905.05094v2, 2019. 
[16] Tamara Kolda. Symmetric orthogonal tensor decomposition is trivial. arXiv preprint arXiv:1503.01375, 2015.

[17] Joseph M. Landsberg. Geometry and complexity theory, volume 169 of Studies in Advanced Mathematics. Cambridge University Press, 2017.

[18] Takanori Maehara and Kazuo Murota. Simultaneous singular value decomposition. Linear Algebra and its Applications, 435(1):106-116, 2011.

[19] Ketan Mulmuley and Milind Sohoni. Geometric complexity theory I: An approach to the $\mathrm{P}$ vs. NP and related problems. SIAM Journal on Computing, 31(2):496-526, 2001.

[20] Ketan Mulmuley and Milind Sohoni. Geometric complexity theory II: Towards explicit obstructions for embeddings among class varieties. SIAM Journal on Computing, 38(3):1175-1206, 2008.

[21] Kevin O'Meara, John Clark, and Charles Vinsonhaler. Advanced topics in linear algebra: weaving matrix problems through the Weyr form. Oxford University Press, 2011.

[22] KC O'Meara and C Vinsonhaler. On approximately simultaneously diagonalizable matrices. Linear Algebra and its Applications, 412(1):39-74, 2006.

[23] Onorato Timothy O'Meara. Introduction to quadratic forms. Springer, 1973.

[24] Elina Robeva. Orthogonal decomposition of symmetric tensors. SIAM Journal on Matrix Analysis and Applications, 37(1):86-102, 2016.

[25] Jussi Salmi, Andreas Richter, and Visa Koivunen. Sequential unfolding SVD for tensors with applications in array signal processing. IEEE Transactions on Signal Processing, 57(12):4719-4733, 2009.

[26] Winfried Scharlau. Quadratic and Hermitian forms. Springer, 1985.

[27] Tong Zhang and Gene Golub. Rank-one approximation to high order tensors. SIAM Journal on Matrix Analysis and Applications, 23(2):534-550, 2001. 